Hydroécol. Appl. (1993) Tome 5 Vol 1, pp. 127-163

\title{
La méthode des micro-habitats: un outil d'aide au choix d'un débit réservé à l'aval des ouvrages hydroélectriques. Expérience d'Electricité de France
}

\author{
The micro-habitat method: tool aid in the choice \\ of discharge best suited to fish life downstream of the \\ hydroelectric schemes. Experience of Electricité de France.
}

\section{Sabaton et J. Miquel*}

Electricité de France - Direction des Etudes et Recherches Département Environnement 6. quai Watier - B.P. 49 - 78401 Chatou Cedex - France

" adresse actuelle: EDF/DTG - 37, rue Diderot - BP. 41 - 38040 Grenoble Cedex

Résumé. - Les ouvrages hydroélectriques s'insèrent dans l'ensemble des ressources en eau d'un bassin. Electricité de France se voit souvent confronté au choix d'un débit à laisser dans la rivière pour préserver l'environnement et satisfaire certains usages. Parmi ceux-ci, l'usage "pêche" ainsi que le souci de protéger l'environnement nécessitent de maintenir une bonne qualité piscicole de la rivière à l'aval.

Le paramètre le plus sensible au débit dans les types de cours d'eau concernés est l'habitat piscicole. La méthode des micro-habitats permet de quantifier cet aspect à l'aide de notes obtenues en reliant les caractéristiques morphodynamiques de la riviere (vitesses, profondeurs, nature des fonds) et "préférences d'habitat " du poisson. On établit alors des graphes donnant l'évolution de ces notes de qualité d'habitat en fonction du débit, et apportant ainsi une aide précieuse au choix d'un débit bien adapté à la vie piscicole.

Ce rapport synthétise les études menées à la Direction des Études et Recherches d'EDF - en collaboration avec plusieurs autres équipes françaises - pour valider et mettre en œuvre cette approche pour les cours d'eau français.

Les résultats des expérimentations faites en France (pêches électriques et avis d'experts) convergent avec celles des chercheurs américains et permettent de confirmer la notion de préférence d'habitat des poissons (représentée par des "courbes de préférence") qui est à la base de la méthode. Grâce au réétalonnage des courbes de préférence de la truite des cours d'eau français, on dispose d'un outil fiable applicable à l'aval de nos ouvrages.

La mise en œuvre pratique de la méthode nécessite une description détaillée du tronçon de rivière et le choix d'unités représentatives qui seront plus particulièrement étudiées. 
Une représentation de la rivière par des zones d'écoulement types - ou faciès - est présentée ici; elle s'avère bien adaptée et assez objective pour un expérimentateur averti. Le nombre de mesures effectives à réaliser pour cerner la morphodynamie du tronçon doit tenir compte de deux impératifs:

- ne pas aboutir à une mise en œuvre trop lourde et trop coûteuse, afin de la rendre applicable au plus grand nombre de sites possible;

- permettre d'obtenir une estimation fiable de l'évolution de la qualité d'habitat du tronçon avec le débit.

En fonction de l'enjeu piscicole lié au site d'étude, il s'agit alors de trouver un bon compromis entre ces deux contraintes.

Le choix effectif d'un débit réservé revient alors au gestionnaire qui doit tenir compte d'objectifs clairement définis. Un effort reste encore à faire au niveau national pour trouver un consensus sur des critères objectifs qui permettront d'utiliser au mieux cet outil de gestion piscicole qu'est la méthode des micro-habitats.

Abstract. - Hydroelectric power stations constitute one element in the overall water resources of a basin. Electricite de France is often confronted with a choice as to the discharge to maintain in a river in order to satisfy certain types of uses. Among these, fishing and environmental concerns are two which require presenvation of the fish resources in the river downstream.

The parameter most sensitive to flow in the different types of stream in question is the fish habitat. The micro-habitat method enables quantification of this aspect on the basis of grades obtained by linking the morphodynamics of the stream (speeds, depths, types of bottom) with fish "habitat preferences". One can then draw up graphs which show the evolution of these habitat quality grades as a function of the discharge, thereby providing invaluable aid in the choice of discharge best suited to fish life.

This report summarizes studies conducted by the EDF Research and Development Division to validate and implement this approach for French streams.

The results of experiments carried out in France (electro-fishing and expert evaluations) converge with those of American researchers, and confirm the notion of fish habitat preference (represented by "probability-of-use curves") which forms the basis of the method. Thanks to our adjustment of the "probability-of-use" curves for trout in French streams, we now have a reliable tool applicable to the waters downstream of our power stations.

Practically, implementation of the method requires a detailed description of the river section and a choice of the representative units to which specific attention will be devoted. A representation of the river in terms of homogeneous flow zones - or facies - is given here. It has proven well suited and objective enough for use by an experienced professional. The number of real measurements which need to be taken to define fully the morphodynamics of the river section must be determined in terms of two imperatives:

- implementation must be neither too complex nor too costly, so as to enable application to as many sites as possible;

- it must provide for good estimation of the evolution in habitat quality as a function of discharge.

Depending on the importance devoted to fish life on the study site, the problem is then to reach a good compromise between these two constraints.

The actual choice of discharge is ultimately that of the water management expert who must take clearly defined set objectives into consideration. There now remains an effort to be made on a national level to reach a consensus with respect to the objective criteria which can enable optimum use of the micro-habitat method as a fish stock management tool. 


\section{INTRODUCTION}

Du débit réservé transitant dans un tronçon de cours d'eau à l'aval d'un aménagement peuvent dépendre plusieurs usages, comme l'irrigation, la production d'eau potable, la pêche, et bien évidemment l'état écologique de la rivière, par le biais de paramètres hydrauliques, thermiques, de qualité chimique et biologique de l'eau.

Une contrainte réglementaire telle que celle imposée par la loi "pêche" peut certainement aider à prendre conscience de l'importance du niveau minimum d'eau à laisser dans l'écosystème, mais le débit réservé est avant tout un élément privilégié de la gestion d'une rivière.

Les grands arbitrages entre usages étant faits, le choix du débit réservé, doit être efficace et bien adapté à chaque cas particulier. II peut fort bien être modulé dans le temps et l'espace. Pour cela, il faut des outils pour mesurer, prévoir et simuler. EDF a contribué à créer de tels outils et sait, depuis plusieurs années, modéliser les paramètres clé d'une rivière: profondeur, vitesse, température, oxygène dissous, biomasse phytoplanctonique.

Les poissons, et plus particulièrement les salmonidés, constituent un indicateur sensible et synthétique du bon état biologique de la rivière. EDF, dont la majorité des aménagements se trouvent sur des secteurs de première catégorie, a cherché à disposer d'une méthode d'analyse et d'un mo- dèle qui lui permettent d'évaluer quantitativement, et objectivement, l'impact du débit résené sur ces espèces. Le choix s'est porté sur la méthode des micro-habitats (Bovee - 1983) développée aux Etats-Unis, qui semble actuellement la mieux adaptée à l'étude précise d'un site.

II a alors fallu la valider sur les rivières françaises et en préciser les conditions d'utilisation, pour éviter d'en faire un usage approximatif ou d'extrapoler les résultats hors de son domaine de validité.

La méthode des micro-habitats ne prétend pas simuler la dynamique des populations piscicoles. Ce n'est qu'associée à d'autres éléments (qualité physicochimique de l'eau notamment, ressources biologiques, alevinage, pression de pêche...) qu'elle peut contribuer à apprécier globalement la qualité piscicole d'une rivière. Au moins permet-elle déjà d'évaluer dans quel sens, et avec quelle sensibilité, une modification du débit réservé se répercutera sur la qualité de l'habitat hydraulique du poisson.

Testée sur un certain nombre de sites, tant par EDF que par d'autres équipes françaises, elle constitue une aide à la décision, maintenant opérationnelle et prête à être utilisée de façon plus courante.

Après avoir rappelé les principes de cette méthode, cet article tente de faire une synthèse de près de dix années de travaux effectués à la Direction des Etudes et Recherches d'EDF 
en collaboration avec plusieurs organismes de recherches (Université de Marseille, ENSA Toulouse, CEMAGREF) et de présenter la démarche retenue par les équipes d'EDF lors de son application.

\section{LA MÉTHODE DES MICRO-HABITATS : RAPPEL ET VALIDATION}

\section{II.1 Principe de la méthode}

Le principe de la méthode consiste à calculer, pour une portion de cours d'eau et un débit donné, une note quantifiant la qualité d'habitat du secteur étudié pour une espèce donnée.

Cette note prend en compte les exigences des populations de poissons vis-à-vis de paramètres morphodynamiques jugés représentatifs de l'habitat: la profondeur, la vitesse, la nature des fonds et éventuellement la température. En effet, on considère qu'une espèce, à un stade de vie donné, aura une préférence pour un type d'habitat qui correspond aux conditions les plus favorables à son développement, mais qu'elle peut se contenter de conditions moins favorables.

La méthode consiste à quantifier ces préférences pour les différents paramètres morphodynamiques qui constituent un habitat, à l'aide d'un indice allant de 1 à 0 selon que les conditions sont plus ou moins satisfaisantes pour l'espèce considérée. Ces indices, quantifiant les préfé- rences des poissons, sont résumés à l'aide de "courbes de préférence d'habitat" (dites aussi "courbes de preferenda ") estimées par espèce, à un stade de développement donné, pour les différents paramètres retenus pour caractériser l'habitat. IIs seront utilisés pour noter la qualité d'habitat du tronçon de rivière étudiée.

La figure 1 présente les courbes proposées par Bovee pour la "brown trout " à ses différents stades de vie.

Le tronçon de rivière à étudier est découpé en éléments homogènes du point de vue de l'habitat. A chacune de ces aires est affectée, pour un débit donné, la valeur des paramètres d'habitat retenus. On calcule alors pour un stade donné et à l'aide des courbes précédentes, comme l'indique la figure 2, la note de préférence d'habitat de chaque aire puis deux notes de capacité d'accueil du tronçon entier: la valeur d'habitat et la surface pondérée utile.

\section{Remarque}

La qualité d'habitat piscicole d'un site est en fait habituellement quantifiée à l'aide de la surface pondérée utile. Cet indice prend simultanément en compte les aspects "qualité" et "quantité " de l'habitat disponible.

Un deuxième indice a également été défini: la valeur d'habitat. II privilégie l'aspect qualitatif puisqu'il n'intègre pas la quantité d'eau présente dans la rivière.

Dans la mesure où l'objectif de la loi pêche n'impose pas de rechercher 

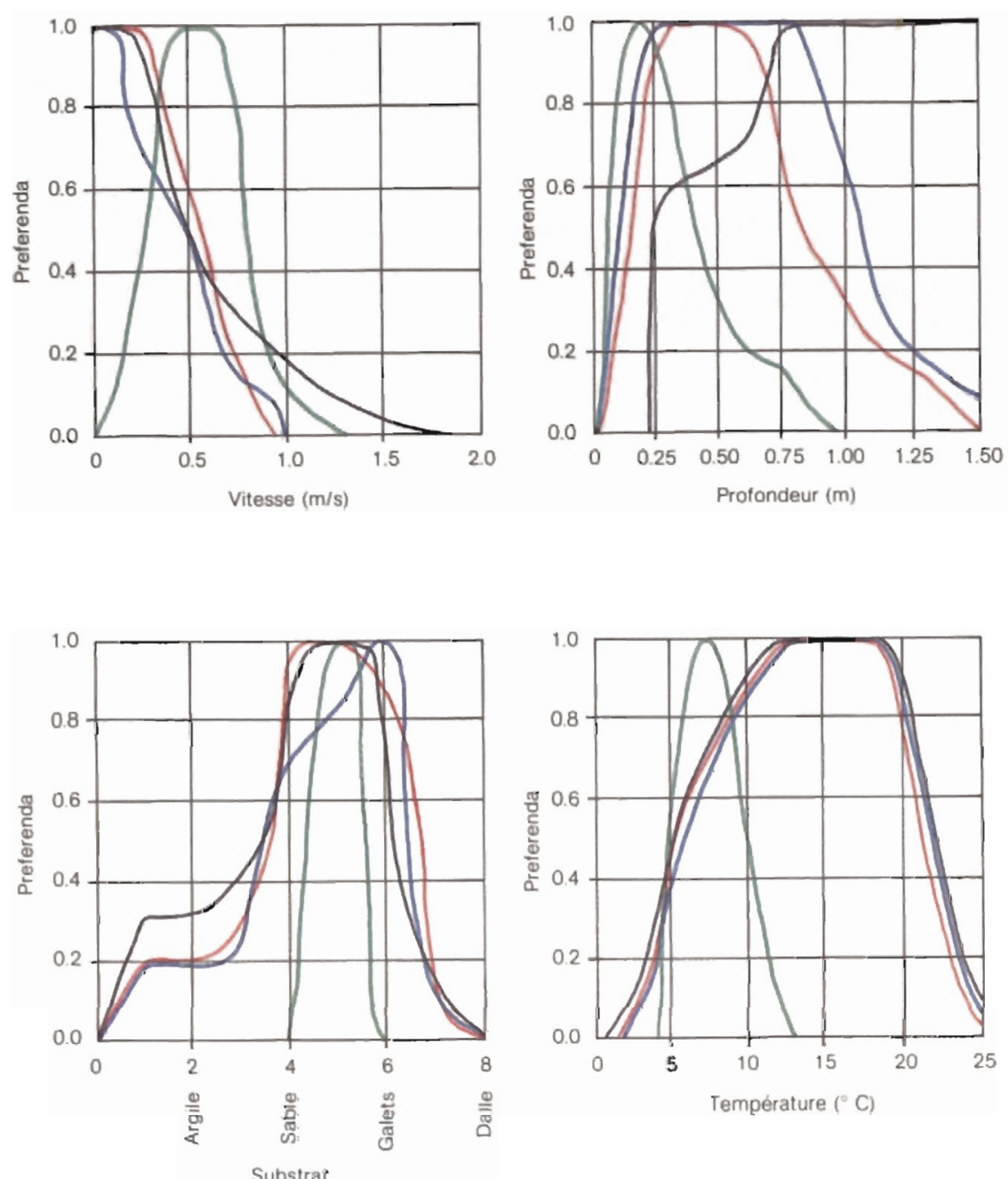

\section{Stade alevin}

Stade juvênile

Stade frai

Stade adulte

Fig. 1. - Courbes de préférence d'habitat de la "brown trout " aux stades adulte, juvénile, alevin et frai, selon Bovee.

Fig. 1. - Probability-of-use curves for the various life stages of brown trout: adult, juvenile, spawning and fry - from Bovee. 
Dans un trónçoń, plusieurs AIRES homogènes $\mathrm{A}_{i}$ - pour un débir $\mathrm{Q}_{j}$ :

\begin{tabular}{|c|c|c|c|c|}
\hline Paramètres & AIRE $A_{1}$ & & AIRE $A_{i}$ & AIRE $A_{n}$ \\
\hline Prolondeur & $\mathrm{Hi}_{\mathrm{i}}^{1}$ & & $H_{j}^{i}$ & $H_{j}^{n}$ \\
\hline Vilesse & $v_{j}^{1}$ & & $v_{j}^{i}$ & $v_{i}^{n}$ \\
\hline Substrat & $s_{i}^{1}$ & & $s_{j}^{i}$ & $s_{i}^{n}$ \\
\hline
\end{tabular}

Détermination des preferenda dhabitat $\mathrm{P}_{\mathrm{Y}}, \mathrm{P}_{\mathrm{h}}, \mathrm{P}_{\mathrm{S}}$. correspondant à la valeur des paramètres pour ce débil à l'aide des courbes de prelerenda dhabilat

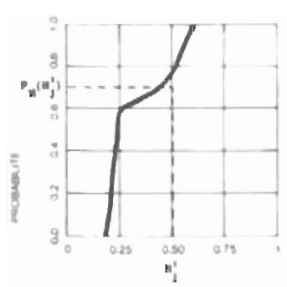

maromofua ind

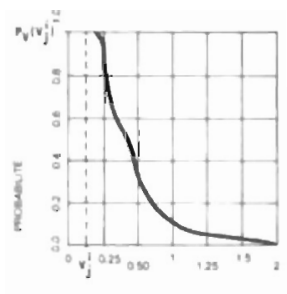

xTtesse im

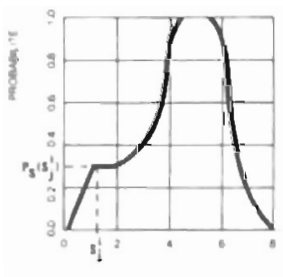

sastar

Calcul d'une evaleur de préterence d'habilat = de chaque aire $A_{i}$ - pour un débit $Q_{i}$ :

$$
\Psi_{i}^{i}=P_{v}\left(V_{i}^{i}\right) \times P_{h}\left(H_{i}\right) \times P_{s}\left(S_{i}^{i}\right)
$$

Echelle :
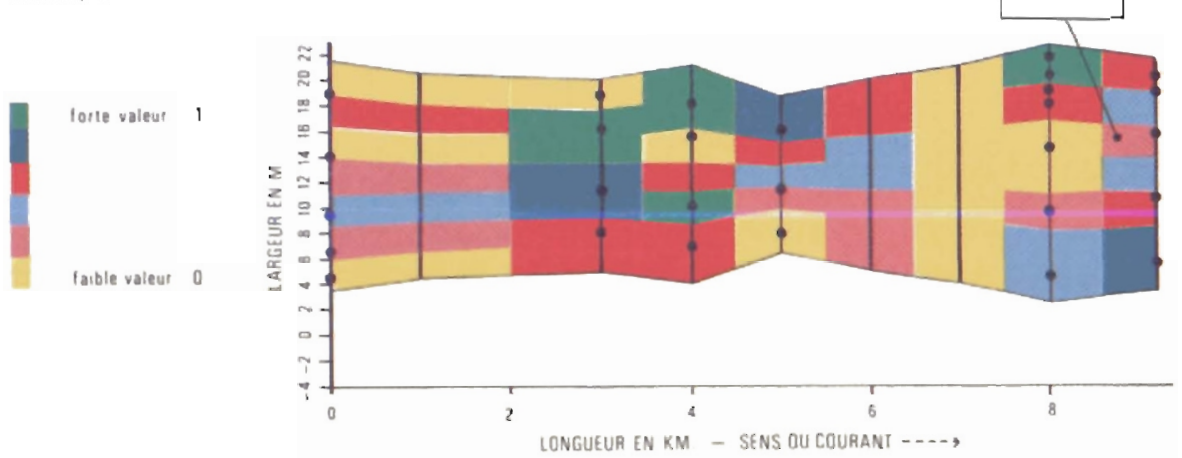

Pour un tronçon total : $\quad$ on calcule 2 indices

$$
\text { SPU }=\sum A_{i} \Psi_{i}^{i} \quad V H A_{j}=\frac{\sum_{i} A_{i} \Psi_{j}}{A}
$$

A : aire totale du tronçon

"Surlace pondérée utile " du tronçon pour un débil $\mathrm{Q}_{\mathrm{j}}$

-Valeur d'habilat - du tronçon pour un débit $\mathrm{Q}_{\mathrm{i}}$

Fig. 2. - Estimation des notes de qualité d'habitat par la méthode des micro-habitats pour un tronçon constitué d’aires homogènes $A_{i}$ et pour un débit $Q$.

Fig. 2. - Estimating quality habitat value through the Instream Flow Incremental Methodology for a reach of river divide into homogeneous cells $A_{i}$ and for a discharge $Q_{j}$. 
une quantité maximale de poissons, nous pensions initialement que la valeur d'habitat - indice qualitatif pourrait permettre à elle seule d'y répondre (garantir la vie et la reproduction des espèces). L'étude des différents sites que nous avons effectuée depuis lors nous a montré qu'elle était tout à fait insulfisante. En particulier, pour les sites de faible module, des valeurs d'habitat peuvent paraitre satisfaisantes pour certains débits alors même que les quantités d'eau correspondantes disponibles dans la rivière ne sont pas suffisantes pour permettre le développement des populations. Le choix d'un débit réservé doit donc dans tous les cas correspondre à un débit pour lequel les valeurs prises par les deux indices se situent simultanément dans une gamme convenable.

\section{II.2 Validation de la méthode pour les rivières françaises}

Les courbes proposées par Bovee pour la "brown trout" ou truite fario ont été tracées à partir de nombreuses observations effectuées sur des cours d'eau américains. Une première étape était de valider ces courbes pour les souches des rivières françaises. Un grand nombre d'expérimentations ont été menées sur des rivières de première catégorie du SudOuest de la France. Dans ces rivières, variées du point de vue morphodynamique, la présence de truites dans des habitats potentiels a été déterminée soit par pêches électriques (par électrodes lancées au hasard, par électrodes fixes - Belaud et al., 1989), soit par avis d'experts (Chaveroche et al., 1989). En croisant ces résultats avec les valeurs des paramètres morphodynamiques mesurés aux lieux explorés, on a ainsi pu construire deux nouvelles séries de courbes de preferenda d'habitat.

La figure 3 permet la comparaison des différentes courbes actuellement disponibles pour les paramètres vitesse et profondeur.

Les courbes de preferenda de vitesse et de profondeur tracées à partir de nos expérimentations sont très comparables à celles proposées par Bovee aux quelques différences près suivantes:

Pour la profondeur, les preferenda estimés par Bovee pour le stade adulte semblent trop faibles pour les valeurs inférieures à $25 \mathrm{~cm}$ au regard de nos estimations. Une étude effectuée par l'équipe du CEMAGREF Lyon (CEMAGREF, 1988) a par ailleurs montré que la relation "biomasse en truites - valeur d'habitat" était meilleure lorsque cette dernière était calculée à partir d'une courbe de préférence proche de celle proposée par les experts.

Pour le paramètre vitesse, une bonne concordance existe entre les courbes de Bovee et celles obtenues à partir des campagnes de pêche; on note par contre, pour la courbe obtenue avec les experts, un net décalage des preferenda vers les vitesses moyennes pour l'adulte, et une décroissance moins rapide des preferenda au niveau de ces mêmes gammes de valeurs pour l'alevin et le 

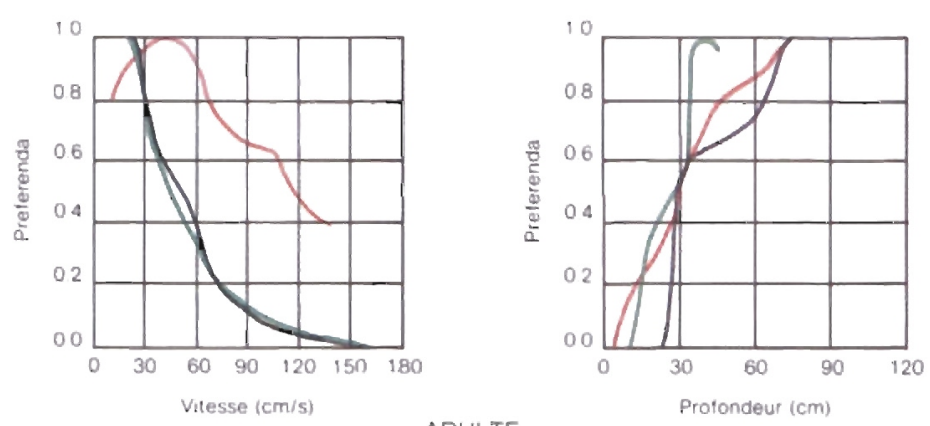

ADULTE
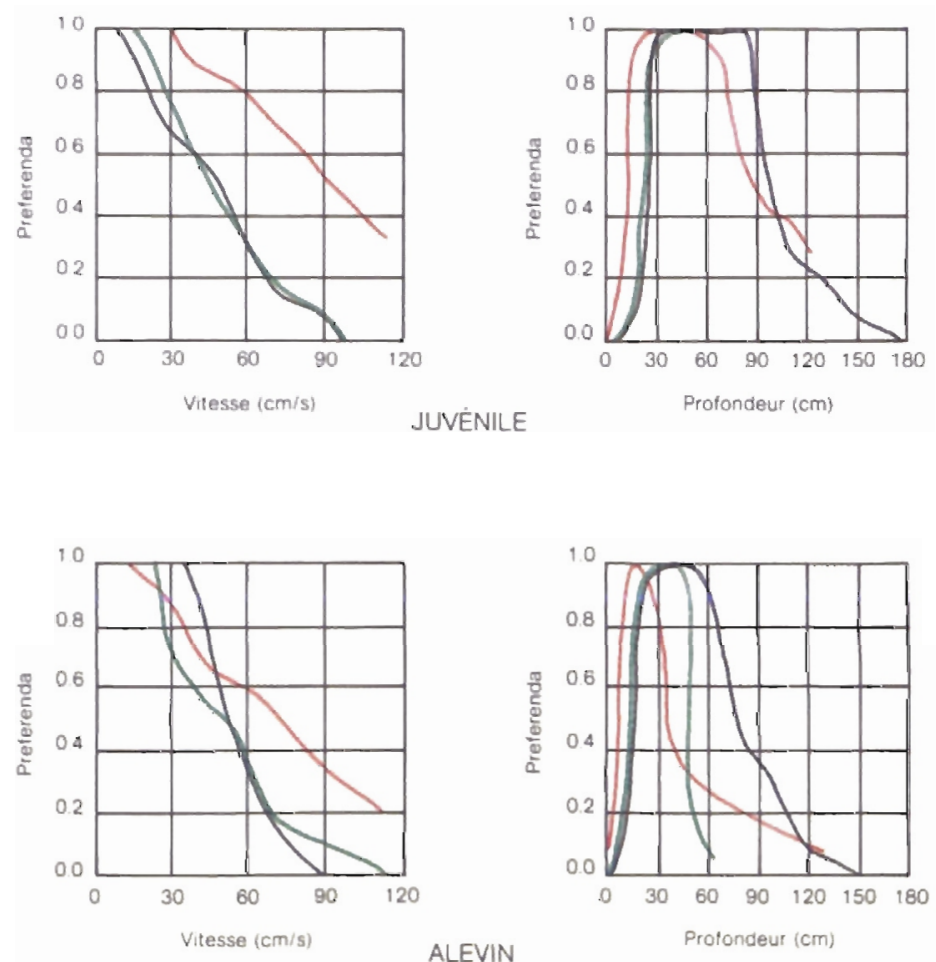

ALEVIN

Selon Bovee

Pêches électriques

Experts

Fig. 3. - Comparaison des courbes obtenues par différentes expérimentations.

Fig. 3. - Comparison between curves constructed from various experiments. 
juvénile. Ce décalage est-il dû à un biais systématique lors des pêches (poissons se réfugiant dans des zones plus calmes si, malgré les précautions prises, les truites ont été "dérangées") ou à la difficulté pour les experts de bien appréhender visuellement un champ de vitesse?

La grande similitude obtenue entre nos pêches par électrodes fixes et les expérimentations américaines nous incitent à conserver les courbes proposées par Bovee pour ce paramètre vitesse.

En ce qui concerne le paramètre substrat le maximum des courbes obtenues par pêche électrique se situe pour les "gros blocs", pour tous les stades (figure 4 pour la truite adulte). Ce résultat diverge de celui donné par Bovee, où ce type de granulométrie correspond à des valeurs de preferenda faibles. Lors des pêches, il est en fait apparu que le substrat "gros bloc" ne semblait pas favorable en lui-même à la présence du poisson: il ne l'est que s'il peut constituer une cache. Un gros bloc sans possibilité de refuge ne constitue pas un substrat très préférentiel. Etant donné l'importance de ce facteur "abri", il serait intéressant d'essayer de l'intégrer directement dans la note d'habitat (qui tiendrait compte alors des blocs "caches" mais également des abris sous berge). II faut toutefois noter que des corrélations biomassevaleur d'habitat effectuées par le CEMAGREF sur huit rivières françaises se sont révélées plus importantes en attribuant au gros bloc une valeur du preferenda plus importante que celle proposée par Bovee.

Malgré ces quelques différences, la cohérence entre les différentes courbes obtenues confirme et accrédite la notion de preferenda et le bien-fondé de la méthode.

En conclusion, tenant compte de toutes ces observations, et dans l'état actuel des connaissances, nous em-

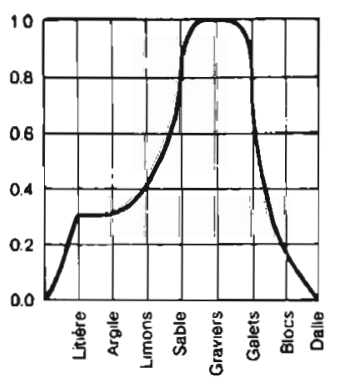

Code Boveo

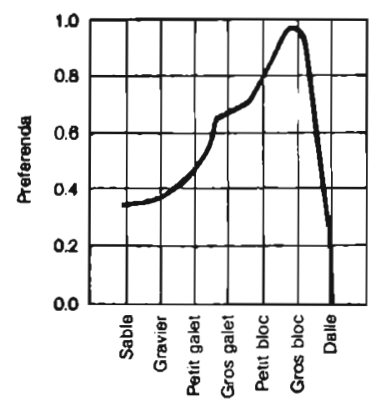

Codo DER

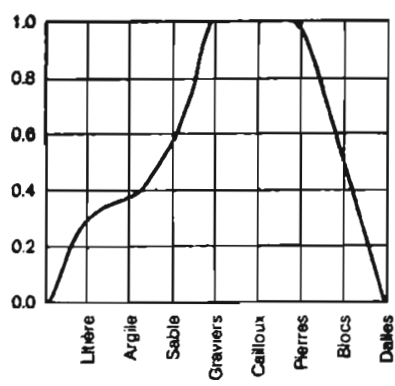

Codo CEMUGREF

Fig. 4. - Courbes de preferenda d'habitat disponibles pour le paramètre substrat truite fario adulte.

Fig. 4. - Available substrate Probability-of-use curves for adult brown trout. 
ployons finalement les courbes de preferenda suivantes:

- courbe tracée par les experts pour la truite adulte vis-à-vis du paramètre profondeur (figure 5);

- courbe proposée par le CEMAGREF (Fragnoud, 1987) pour la truite adulte vis-à-vis du paramètre substrat (figure 5);

- courbes tracées par Bovee pour les autres paramètres ou autres stades.

\section{II.3 Les autres paramètres à prendre en compte pour évaluer la qualité d'habitat}

En plus du paramètre "cache", d'autres paramètres ou aspects de l'habitat doivent être examinés. A notre avis, il est prématuré ou mal adapté de les intégrer dans la méthode sous forme de courbes de preferenda: il est toutefois indispensable de les appréhender à l'aide de mesures, d'avis d'experts et de modèles si nécessaire.

\section{II.3.1 La température}

Des courbes de preferenda ont été proposées par Bovee pour le paramètre température.

Les valeurs de température suivent des variations journalières et saisonnières non liées de manière biunivoque aux valeurs de débit. Pour intégrer ce paramètre à la méthode des micro-habitats, on pressent bien qu'une simple mesure des températures des rivières échantillonnées n'est pas suffisante pour synthétiser les préférences du poisson. Les variations spatiales et temporelles de températures sont certainement des facteurs plus importants à prendre en compte.

On peut également imaginer que lors d'épisodes courts d'élévation importante de la température, le poisson puisse aller vers des zones de refuge
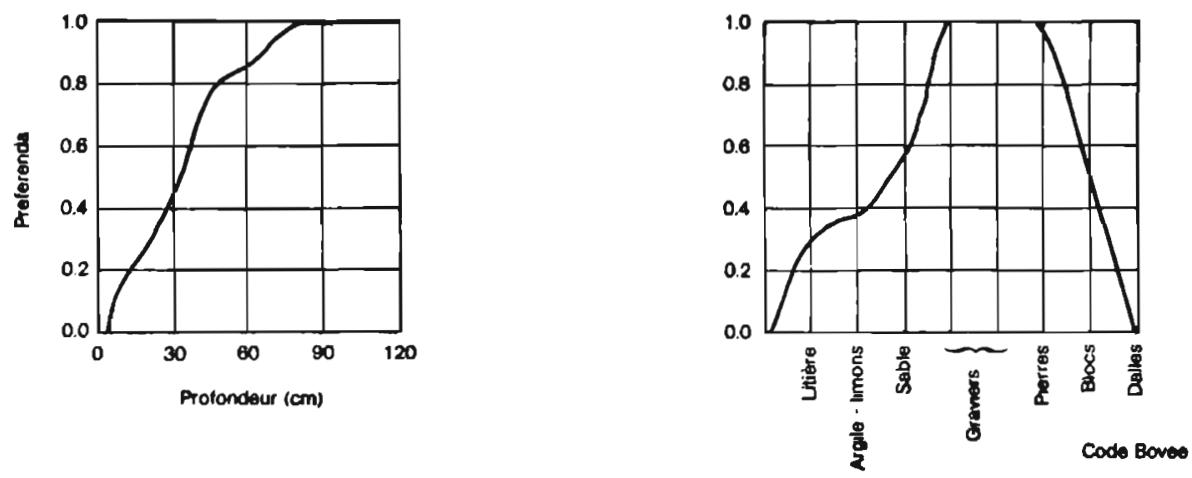

Fig. 5. - Courbes de preferenda actuellement retenues par EDF/DER pour la truite fario adulte (en remplacement de celles proposees par Bovee) pour les parametres profondeur et substrat.

Fig. 5. - Probability-of-use cures used by EDF/DER for adult brown trout, for depth and substrate. 
locales plus fraîches. Une étude plus approfondie du comportement de la truite est donc nécessaire pour déterminer précisément les critères à retenir (seuils critiques à ne pas dépasser, durées maximales d'exposition à des températures supérieures à ces seuils, gradients thermiques, etc...).

A un niveau plus global, la dynamique des populations de diverses rivières de régimes thermiques très variés devrait aider à comprendre l'influence de ces derniers sur les peuplements.

En attendant, il s'agira de vérifier, lors de l'étude d'un site, que les valeurs de température dans les divers tronçons restent, pour les débits envisagés, dans une gamme compatible avec la vie des espèces de poissons concernées.

Dans certains cas critiques, un modèle thermique peut devoir être mis en œuvre pour apprécier l'influence du débit sur le régime thermique du cours d'eau.

\section{II.3.2 Autres paramètres de quali- té d'eau}

Comme pour le paramètre température, il s'agira de vérifier que les différents paramètres de qualité d'eau restent dans des gammes acceptables. C'est généralement le cas pour les aménagements hydrauliques situés en amont des rivières.

Une campagne de mesures et/ou un suivi annuel permet en général de faire cette vérification. II faut cependant noter que la modélisation de l'oxygène dissous peut être précieuse, comme celle de la température.

\section{II.3.3 La valeur trophique}

Les paramètres retenus (vitesse, profondeur, nature du substrat) influent localement sur la productivité du milieu, et donc sur la nourriture disponible au voisinage de l'habitat considéré.

On doit cependant s'interroger pour connaitre dans quelle mesure le facteur trophique est implicitement pris en compte.

La dérive issue du barrage, des zones amont et des affluents, qui dépend certainement du débit, est indépendante des conditions locales prises en compte dans la méthode des micro-habitats. On peut par contre supposer que le benthos et la faune de dérive - qui est directement issue de ce dernier - sont pris en considération lors de l'étude d'un micro-habitat. Quant aux apports exogènes qui constituent la part aérienne de la dérive, ils sont difficilement quantifiables et surtout indépendants du débit.

\section{II.3.4 Habitat lié au frai}

Bovee a établi des courbes de preferenda caractérisant les zones de frai. Les diverses mesures hydrauliques effectuées sur des frayères de truites dans différents cours d'eau en France coïncident parfaitement avec ces preferenda.

L'utilisation de la méthode des micro-habitats pour cette fonction vitale qu'est la reproduction doit être cependant faite avec précaution.

Au niveau de l'application même de la méthode il s'agit d'être très vigilant. II faut en effet noter que les zones 
propices au frai sont très localisées et que l'on risque de mal les représenter lors du choix des secteurs caractéristiques. Une reconnaissance préalable des zones de frai potentielles est donc souvent nécessaire.

\section{PRINCIPE DE MISE EN CEUVRE}

Le calcul des indicateurs de qualité d'habitat suppose de connaître en tout point, et pour tout débit, les profondeurs et vitesses de l'eau et le substrat.

Ceci est bien évidemment impossible en toute rigueur: pour un tronçon de 20 mètres de large sur $10 \mathrm{~km}$ de long, cela représenterait quelques 200000 points de mesures... dont la précision dépendra de l'effort de mesures et de calcul consenti.

\section{III.1 Les différents types d'écoulement}

La rivière est une succession de "faciès", qui correspondent à des configurations morphodynamiques différentes dues aux variations de pente, de largeur, de granulométrie, de végétation, et donc de vitesse, de profondeur, de turbulence, etc...

Pour pouvoir définir objectivement ces faciès, il a fallu les décrire, les mesurer, les classer à partir d'un grand échantillon, et finalement en produire une première liste qui est la suivante:

Tableau I. - Classification des différents types de faciès.

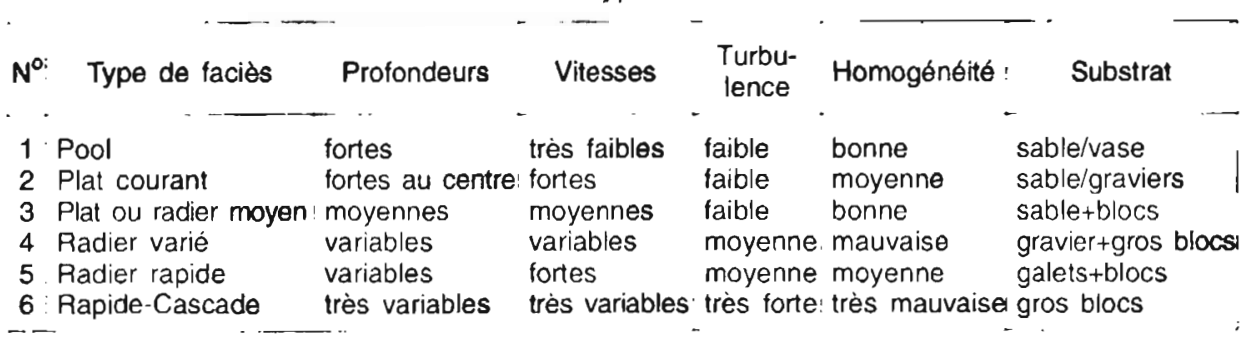

On sera le plus souvent amené à n'examiner que des secteurs représentatifs; ceci suppose de définir préalablement des types d'écoulement dans la rivière. L'estimation des paramètres d'habitat se fera à l'aide de mesures et/ou de modèles hydrauliques. On obtiendra alors une estimation de la qualité d'habitat de la rivière
Cette classification est très synthétique, mais suffit déjà pour découper la rivière en zones bien typées.

Cependant, il est tout à fait possible de l'affiner, soit en détaillant chacune des familles ci-dessus (Chaveroche, 1990; Sabaton, 1991) soit en utilisant une typologie traduisant la dynamique fluviale (Malavoi, 1989). 
L'important est que l'identification in situ des faciès reste fiable.

\section{III.2 Mesures et Modélisation hy- draulique}

Trois approches sont en fait possibles:

- modélisation hydraulique classique complète de l'ensemble du tronçon;

- mesures en quelques secteurs représentatifs et éventuellement modélisation hydraulique simplifiée;

- mesures et modélisation hydraulique classique en quelques secteurs représentatifs.

\section{III.2.1 Modélisation hydraulique classique complète de l'ensem- ble du tronçon}

Dans le cas où la rivière est suffisamment régulière, où la pente n'est pas excessive et où la granulométrie n'est pas importante devant les profondeurs, un modèle hydraulique filaire permanent suffit pour connaître la cote de la ligne d'eau et la vitesse moyenne en toute section transversale (ou transect) de l'ensemble du tronçon pour un débit donné (Cherubini et al., 1992).

Comme le calcul d'habitat s'effectue à l'échelle locale (le $\left.\mathrm{m}^{2}\right)$, il faut passer de ces caractéristiques moyennes d'une section à des caractéristiques locales d'habitat.

La méthode adoptée consiste à découper chaque section en $\mathrm{N}$ tranches verticales sur lesquelles on mesure, pour un débit $Q^{*}$, la profondeur $h_{i}^{*}$ et la vitesse $v_{i}^{*}$, (moyenne sur la tranche verticale i).

Connaissant la côte $Z^{\star}$ de la ligne d'eau, la topographie permet de calculer la largeur $L^{*}$ et la surface mouillée $S^{*}$, associées à $Q^{*}$; on en déduit alors les profondeur et vitesse moyennes de la section:

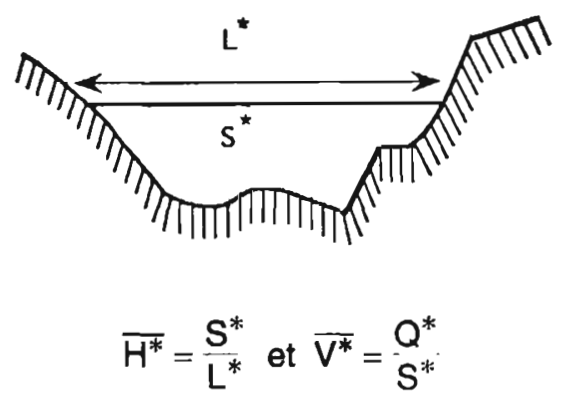

Pour un autre débit $Q$, des mesures ne seront plus forcément nécessaires: le modèle hydraulique fournit la cote $Z$ et par suite les profondeur et vitesse moyennes de la section; le découpage de cette section en $\mathrm{N}$ tranches verticales associé au débit $Q$ sera obtenu à partir du précédent découpage à l'aide d'une homothétie de rapport $\frac{L}{L^{*}}$ :

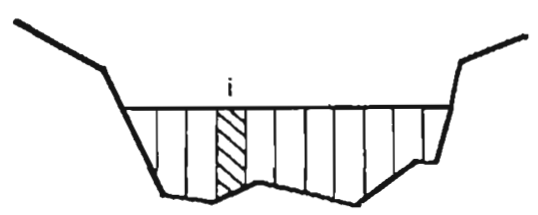

Débit Q*

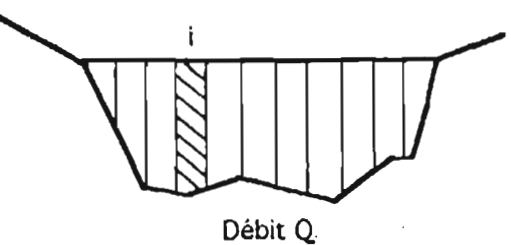

Débit $Q$. 
Pour calculer les valeurs $h_{i}$ et $v_{i}$ associées à $Q$, on utilise les relations de proportionnalité suivantes:

$$
h_{i}=h_{i}^{*} \frac{\bar{H}}{H^{*}} \text { et } v_{i}=v_{i}^{*} \frac{\bar{V}}{V^{*}}
$$

(le débit $q_{i}$ passant dans la tranche $\mathrm{i}$ est alors supposé varier proportionnellement au débit total: $q_{1}=q_{i}^{*} \frac{Q}{Q^{*}}$ )

II ne s'agit évidemment que d'une approximation qui nous a paru compatible avec les précisions globales attendues, mais qui pourrait être perfectionnée en introduisant quelques hypothèses sur les pertes de charges locales.

L'intérêt de cette approche est de ne nécessiter qu'une ou deux lignes d'eau mesurées. Elle est donc applicable aussi bien aux rivières régulées, qu'aux rivières non régulées sur lesquelles il est impossible de maintenir un débit fixé à l'avance.

Cette approche est également la plus précise lorsque les hypothèses d'écoulement sont vérifiées et lorsque les données topographiques sont denses.

II faut bien reconnaître que, malheureusement, rares sont les cas où ces deux conditions sont remplies. De plus, l'information topographique qu'elle requiert est très coûteuse.

\section{III.2.2 Mesures en quelques sec- teurs représentatifs et modélisa- tion hydraulique simplifiée}

Les inconvénients mentionnés ci-dessus vont naturellement conduire les utilisateurs à réduire le volume des mesures à effectuer en choisissant quelques secteurs caractéristiques (faciès ou ensembles de faciès) pour représenter au mieux le tronçon. Ces faciès sont généralement décrits par des sections transversales dont l'évolution avec le débit pourra être définie à l'aide de mesures.

Lorsque le problème du débit réservé se pose pour un site existant, le tronçon de rivière est régulé; il est alors possible de maintenir différents débits fixés à l'avance, et de mesurer pour chacun d'eux les caractéristiques hydrauliques des secteurs représentatifs des différentes parties du tronçon.

Si les débits explorés sont assez nombreux, une simple interpolation dans l'espace et en débit suffit pour obtenir une cartographie de la qualité d'habitat de la rivière en fonction du débit.

Si les mesures n'ont pas été effectuées pour un nombre suffisant de débits, on peut alors, pour chaque transect, estimer à l'aide d'un modèle hydraulique simplifié, l'évolution des profondeur et vitesse moyennes à l'aide des formules du type:

$$
\begin{aligned}
& \bar{H}=a_{h} Q^{b_{n}} \\
& \bar{V}=a_{v} Q^{b_{v}}
\end{aligned}
$$

où $a_{h}, b_{h}, a_{v}$ et $b_{v}$ sont estimés à partir des mesures disponibles.

La reconstitution des hauteurs et vitesses locales $h_{i}$ et $v_{i}$ s'effectue alors en utilisant la relation de proportionnalité présentée au paragraphe précédent. On en déduit alors l'évolution des notes d'habitat des transects avec 
le débit, puis par extrapolation dans l'espace, celle du tronçon étudié.

\section{III.2.3 Mesures et modélisation hydraulique classique en quel- ques secteurs représentatifs}

Lorsqu'une modélisation complète de la rivière semble trop lourde ou bien lorsque l'on ne peut pas maîtriser les débits amont, une méthode mixte peut-être utilisée. Comme précédemment, elle consiste à choisir des secteurs représentatifs constitués ici de faciès accolés successifs.

On réalise alors une modélisation hydraulique classique locale à partir d'une ou deux lignes d'eau très détaillées.

Cette méthode, intéressante pour une rivière non régulée, est notamment employée par le CEMAGREF. Le modèle hydraulique utilisé est un modèle filaire perfectionné de façon à prendre en compte les pertes de charges locales (Baume et al., 1984; Souchon et al., 1989).

\section{III.2.4 Synthèse: quelle approche utiliser?}

Lorsque la rivière s'y prête et que l'effort de mesure est acceptable, l'idéal est d'employer la méthode par modélisation complète de l'ensemble du tronçon.

En pratique, cela est rarement possible, et c'est en fait la méthode par mesures et modélisation simplifiée que nous utilisons le plus fréquemment à l'aval des barrages EDF. Nous présenterons plus en détail au chapitre IV sa mise en œuvre pratique.
La troisième méthode présentée, bien adaptée aux rivières non régulées, soulève en fait les mêmes questions que l'approche précédente, en particulier la représentativité des secteurs caractéristiques étudiés et la précision des résultats obtenus. Elle peut pratiquement suivre le même schéma d'application en remplaçant l'extrapolation simplifiée des grandeurs hydrauliques avec le débit par la modé- lisation hydraulique classique.

\section{III.3 Précision des résultats obtenus}

La valeur des estimations des différentes notes de qualité calculées est liée à plusieurs types d'incertitudes:

- incertitudes dues à la représentation du tronçon total par les seules zones précisément étudiées;

- incertitudes liées aux modèles hydrauliques mis en œuvre;

- incertitudes liées au nombre de mesures effectuées pour étudier chacun des secteurs représentatifs choisis.

\section{III.3.1 Représentativité des zones étudiées}

L'incertitude liée à la représentativité des zones mesurées est difficilement cernable: leur nombre optimal et le choix effectif de ces zones dépendent de l'hétérogénéité des écoulements de la rivière mais également du jugement de l'expérimentateur. La phase 
de reconnaissance préalable du tronçon à étudier est donc primordiale.

\section{III.3.2 Modèles hydrauliques}

Après diverses études de sensibilité et en particulier une étude effectuée sur l'Orne (Courot, 1989), on a estimé à environ $20 \%$ l'ordre de grandeur de la précision sur les indices de qualité d'habitat due à l'utilisation de modèles hydrauliques pour reconstituer les vitesses et profondeurs des micro-habitats caractéristiques.

\section{III.3.3 Nombres de mesures effec- tuées dans les zones représenta- tives}

L'étude détaillée de l'évolution des habitats avec le débit est faite dans des faciès caractéristiques préalablement définis $(\S I I I, 2)$.

Des mesures et/ou modélisations hydrauliques ne peuvent être effectuées en tout point de ces faciès. Une discrétisation de chaque faciès est alors nécessaire.

Lorsqu'il s'agit de mesurer les vitesses et profondeurs pour plusieurs débits lâchés deux protocoles sont possibles:

- tirage au sort d'un semis de points sur lesquelles les mesures seront effectuées. Le hasard peut-être "affiné " en tenant compte des caractéristiques du faciès (en prenant par exemple $1 / 3$ de points sur chacune des bordures et 1/3 au milieu de la rivière);

- choix de transects représentatifs: les mesures sont alors faites le long de ces transects. II faut noter que lorsqu'une modélisation est nécessaire (classique ou simplifiée), cette approche par transects est alors indispensable.

Du nombre de mesures effectivement faites dépend la qualité de l'estimation de la note de qualité d'habitat du faciès. Une étude spécifique effectuée sur une rivière des Alpes, la Gyronde (Parent, 1991) a permis de donner un ordre de grandeur de la qualité d'information nécessaire à la bonne représentation d'un type donné d'écoulement.

Le tableau ci-dessous résume les résultats obtenus.

Tableau 2. - Nombre de points ou de transects de mesures conseillés sur un faciès donné suivant son type.

Type de faciès $\quad$ Nombre de transects
Nombre de points aléatoirement choisis

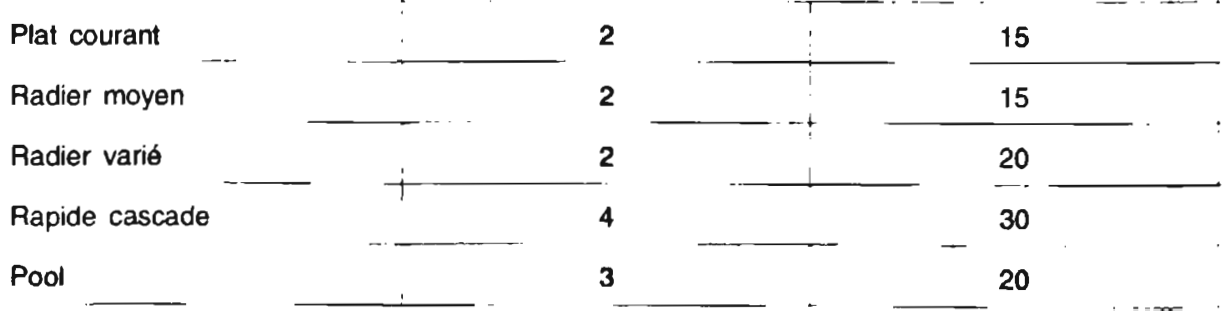




\section{LES PRINCIPALES ÉTAPES DE LA MISE EN CEUVRE}

La mise en œuvre de la méthode des micro-habitats présentée ici est celle utilisée le plus fréquemment par EDF (mesures et/ou modélisation simplifiée). Elle repose sur cinq grandes étapes (schématisée figure 6).

- reconnaissance de la rivière et éventuellement détermination de sous-tronçons;

- choix des secteurs (faciès) représentatifs et estimation des pourcentages de la rivière qu'ils représentent;

- mesures morphodynamiques etou modélisation hydraulique simplifiée au sein de ces secteurs;

- estimation des notes de qualité d'habitat de ces faciès puis extension aux sous-tronçons et au tronçon global;

- analyse de l'ensemble des résultats obtenus en fonction des objectifs de gestion que l'on s'est fixés et proposition d'un débit réservé.

Remarque: seule la $3^{\theta}$ étape est à modifier si l'on doit effectuer une modélisation hydraulique classique au sein des secteurs représentatifs (faciès choisis alors accolés - voir $\S$ III.2.3 et III.2.4).

\section{IV.1 Reconnaissance de la rivière et détermination des sous-tron- çons}

Il s'agit, à l'aide de la classification des faciès choisie (par exemple celle présentée $\S$ III.1), de repérer - et de noter sur une carte détaillée - la succession des différents types de facès rencontrés.

Ce repérage peut s'effectuer à pied en descendant la rivière lorsque les accès aux berges le permettent. L'usage de photos aériennes ou, lorsque cela est possible, d'un hélicoptère et de prises de vue vidéo peut être utile.

L'évolution du profil en long, d'éventuelles disparités amont-aval, ou la présence d'apports intermédiaires, conduisent le plus souvent à découper la rivière en sous-tronçons qui seront étudiés spécifiquement. Dans le cas d'apports intermédiaires, ceux-ci devront être évalués, voire mesurés, en fonction de la saison. Ils viendront s'ajouter dans chacun des sous-tronçons au débit réservé délivré à l'aval de l'aménagement.

\section{IV.2 Choix des faciès représenta- tifs}

Les faciès représentatifs sont choisis au sein de chacun des sous-tronçons d'étude. Leur choix doit permettre d'avoir un échantillon de l'ensemble des faciès suffisamment important pour atténuer l'effet d'échantillonnage. Sa taille dépendra de l'hétérogénéité des écoulements pour un même type de faciès (ou pourra par exemple être amené à considérer deux types de pools...).

La reconnaissance du tronçon étudié permet d'établir le pourcentage de rivière représenté par chacun des faciès représentatifs choisis. Ces pourcentages serviront à pondérer les 


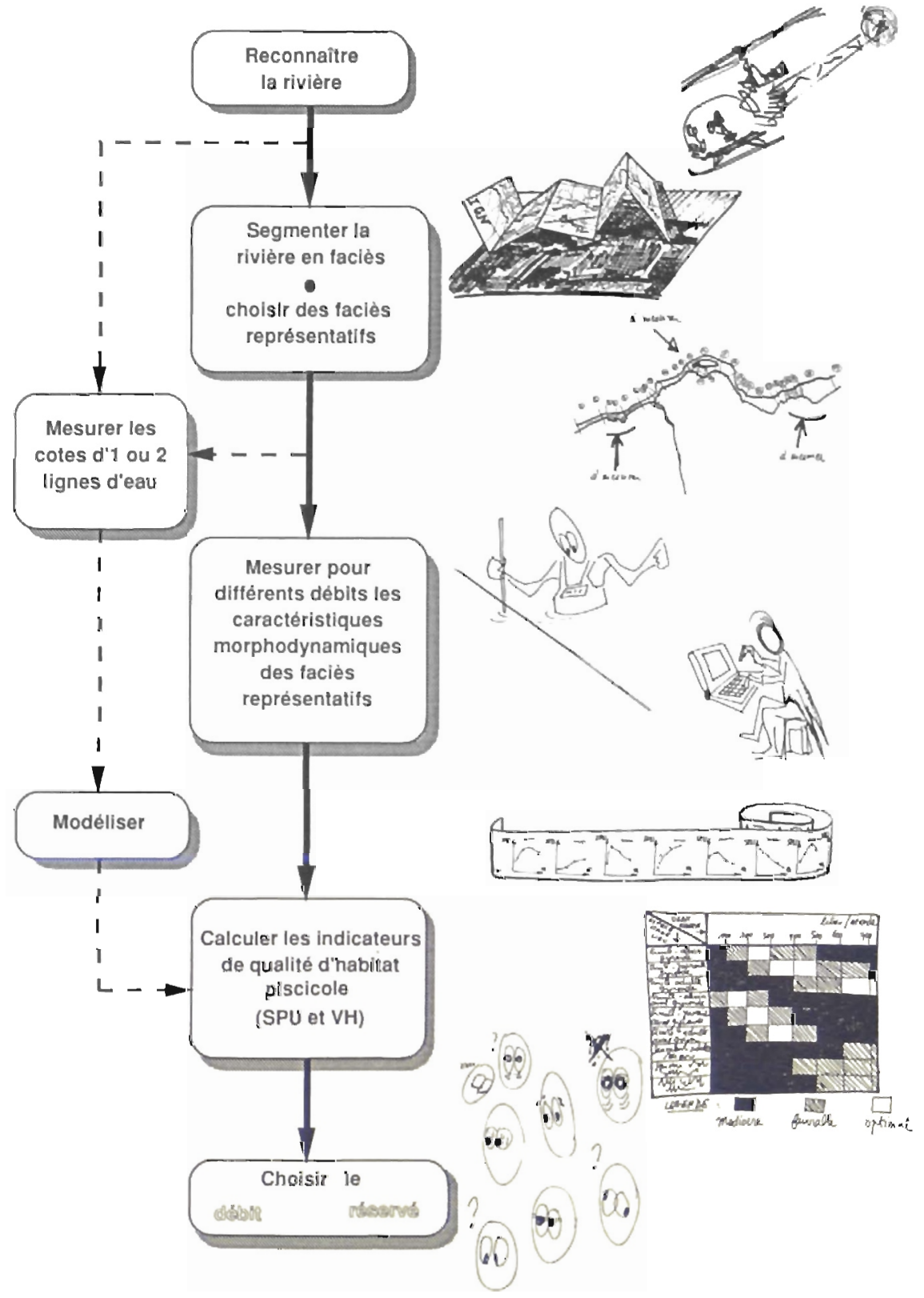

Fig. 6. - Les principales étapes de la mise en œeuvre de la méthode des micro-habitats. Fig. 6. - Main steps in the implementation of the Instream Flow Incremental Methodology. 
notes de qualité d'habitat obtenues pour estimer celles des sous-tronçons et du tronçon global.

Cette pondération basée sur la représentativité linéaire des faciès peut être réajustée en tenant compte de priorités locales (tronçons plus accessibles, zones à privilégier etc...).

\section{IV.3 Mesures morphodynamiques détaillées - modélisation hydrauli- que simplifiée}

Le nombre de mesures est déterminé en tenant compte des types d'écoulement (§ III.3.3).

En ce qui concerne la mesure proprement dite, elle comporte en un point donné:

- la mesure de la profondeur;

- la mesure de la vitesse au 2/3 de la surface (donc $1 / 3$ du fond). Ce niveau n'a pas de justification biologique : c'est en fait un indicateur de l'ensemble de la distribution verticale des vitesses qui s'est avéré statistiquement bien relié à la présence ou à l'absence de poisson;

- la description du substrat, en indiquant les pourcentages de chaque type de substrat observé sur environ $1 \mathrm{~m}^{2}$ autour du point de mesure.

Ces mesures sont faites pour différents débits, maintenus stabilisés grâce au réservoir amont.

II est important d'explorer une gamme de débit suffisamment large (et si possible plus large que celle des débits envisagés) pour limiter les extrapolations.
Si de telles approximations sont nécessaires (nombre de débits mesurés trop faible), on effectue alors, sur chaque transect mesuré, la modélisation hydraulique simplifiée (type $\bar{H}=a_{h} Q^{b_{n}}$ et $\bar{V}=a_{v} Q^{b_{v}}$ ) présentée $\S$ III.2.2 et l'on reconstitue l'évolution des paramètres hydrauliques des transects dans la gamme des débits souhaitée. II faut alors savoir que le risque d'inadéquation du modèle dépend de la complexité de la forme du lit et croît rapidement lorsque l'on s'écarte des débits mesurés.

\section{IV.4 Calcul des notes de qualité d'habitat piscicole}

Les mesures et/ou la modélisation hydraulique simplifiée effectuées permettent alors d'estimer, pour différents débits, les notes de qualité des faciès caractéristiques puis celles du tronçon entier, en intégrant les pourcentages de représentativité des secteurs étudiés (figure 7).

\section{IV.5 Analyse des résultats et choix d'un débit réservé}

\section{IV.5.1 Synthèse des courbes obte- nues}

Les calculs effectués précédemment se visualisent par autant de courbes estimant l'évolution des indicateurs d'habitat avec le débit qu'il y a de stades, voir d'espèces.

Ces courbes sont tracées soit pour l'ensemble de la rivière, soit éventuellement pour quelques zones d'intérêt 
$\Longrightarrow \quad$ Calcul des notes d'habitat quand le faclès est représenté par un ou plusieurs transects Faciès représenté par $\mathrm{N}$ transects :

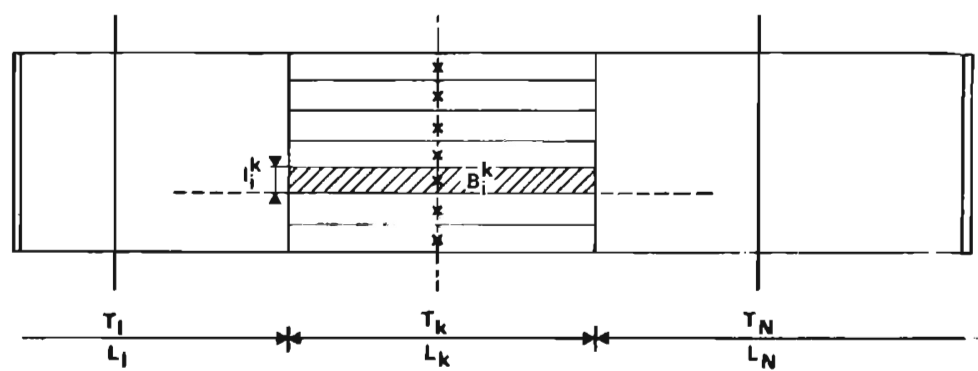

Mesures des paramètres morphodynamiques effectuées aux points $B_{i}^{k}$ pour un débit $Q$<smiles>C=CC=C</smiles>

Valeur d'habitat et surface pondérée utile du facies au debit $Q$ :

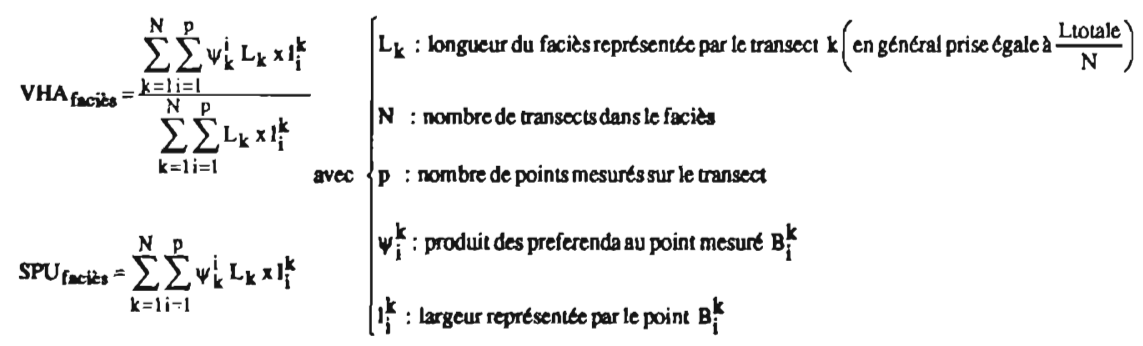

\section{$\Longrightarrow \quad$ Estimation des notes d'habitat du tronçon}

Tronçon représenté par $M$ faciès :

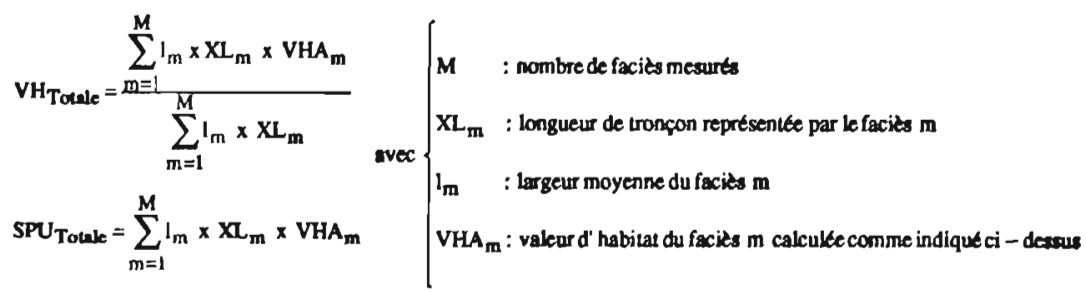

attention : la SPU est donnée ici en $\mathrm{m}^{2}$ utile de l'ensemble du tronçon ; elle s'exprime souvent en $\mathrm{m}^{2}$ par $\mathrm{m}$ ou par $\mathrm{km}$ de rivière.

Fig. 7. - Démarche du calcul des notes de qualité d'habitat d'un tronçon - exemple de faciès représentés par des transects.

Fig. 7. - Computation steps for setting up habitat quality value of a river reach - example of bed patterns represented by cross-sections. 
particulier en fonction d'objectifs locaux spécifiques.

Compte tenu des différentes incertitudes liées aux estimations des notes d'habitat, nous préférons définir pour la lecture des courbes obtenues, trois plages de débits qui correspondent aux qualités d'habitat suivantes:

- indice de qualité compris entre 80 et $100 \%$ du maximum observé sur la gamme de débits étudiés: le débit sera dit "optimum";

- indice de qualité compris entre 50 et $80 \%$ du maximum observé: le débit sera dit "favorable";

- indice de qualité inférieur à $50 \%$ du maximum observé: le débit sera dit "médiocre".

Un tableau comparatif des plages obtenues (comme celui présenté figure 8) peut alors aider à trouver, le cas échéant, les compromis à faire entre les différentes espèces et les différents stades.

Les espèces: un tronçon de rivière peut abriter plusieurs espèces. Faut-il privilégier, par exemple, la truite ou le saumon?

Les stades: pour une même espèce, faut-il privilégier l'adulte, l'alevin? Faut-il favoriser la fonction de reproduction?

Une discussion avec les fédérations de pêche locales permet souvent de définir une ligne de conduite à suivre. L'idéal est parfois une modulation du débit pendant l'année qui permet de tenir compte de l'évolution des différentes stades: on privilégiera par exemple le frai en hiver, les alevins au printemps...

\section{IV.5.2 Le choix d'un débit réservé}

Cartographies, courbes de qualité d'habitat en fonction du débit, et tableau de synthèse obtenus à partir d'une mise en œuvre rigoureuse de la méthode des micro-habitats constituent des éléments précieux pour aider au choix d'un débit réservé.

II faut ajouter à cette information celle concernant d'autres paramètres de l'habitat: la température, l'oxygène, la qualité d'eau, etc. Un minimum de mesures doit être effectué pour garantir qu'aucun paramètre n'est limitant, ce qui rendrait illusoire tout effort sur l'habitat hydraulique.

Une expertise reposant sur les informations quantitatives, et en particulier sur les plages de débit "optimum, favorable ou médiocre" obtenues pour les différents stades, permet en général de définir approximativement trois seuils pour le débit réservé:

- un seuil inférieur au-dessous duquel la qualité piscicole du milieu est compromise;

- un seuil supérieur au delà duquel la qualité piscicole commence à redécroitre;

- un seuil intermédiaire au delà duquel la qualité d'habitat piscicole ne s'accroît plus de façon sensible. En présence de contraintes économiques, il est souhaitable de chercher à se rapprocher au mieux de ce seuil si celui-ci est compatible avec les objectifs fixés. 


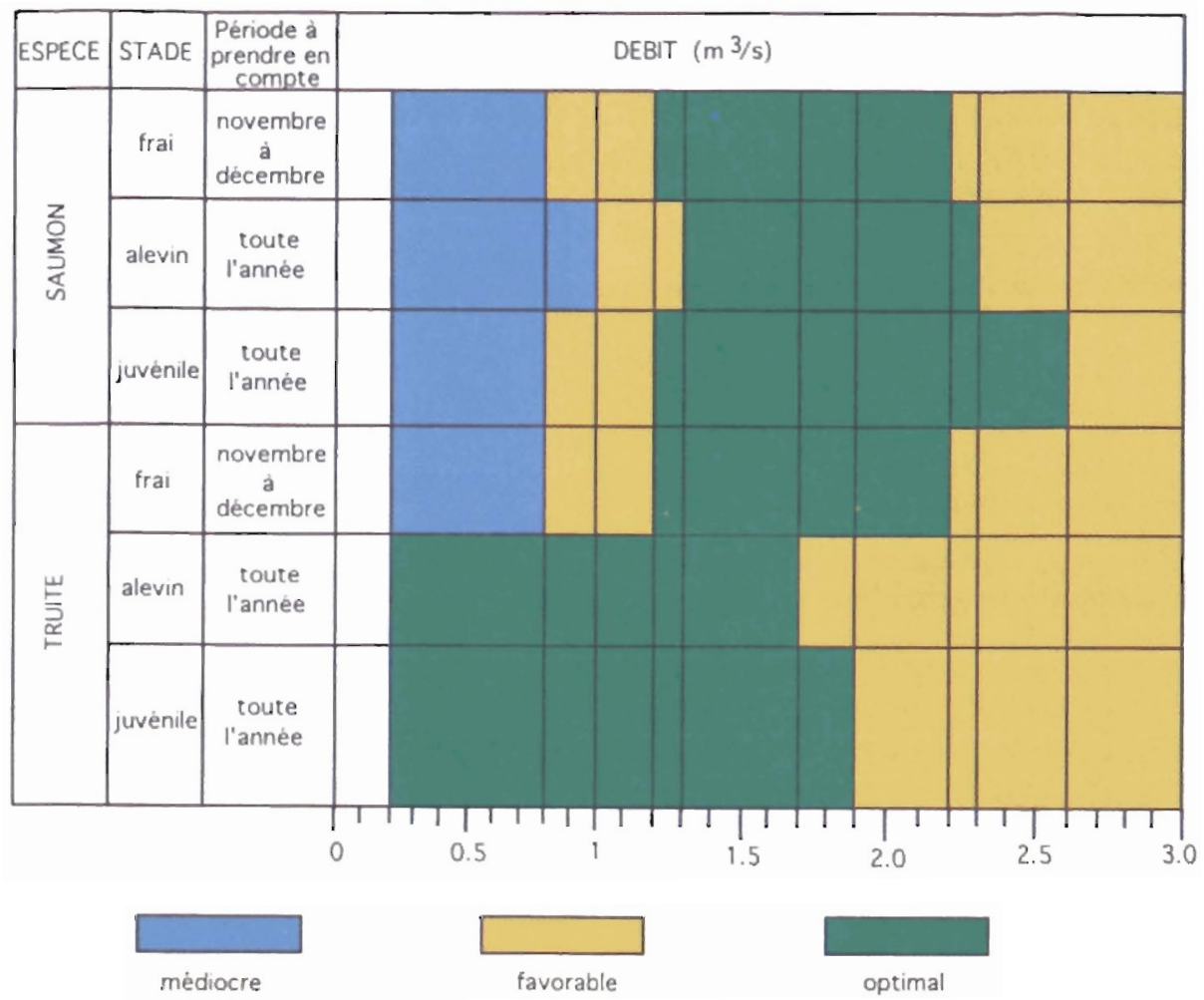

Fig. 8. - Synthèse des résultats obtenus sur l'Orne à l'aval de Rabodanges pour la truite de mer et le saumon.

Fig. 8. - Synthesis of results for the Orne river downstream of Rabodanges reservoir for sea trout and salmon.

\section{$\checkmark$ EXEMPLES}

Plusieurs études de sites nous ont progressivement conduit à retenir le protocole de mise en œuvre décrit précédemment. Ce protocole étant à l'époque en cours d'élaboration, les exemples présentés n'en sont pas des modèles d'application rigoureux.

Toutefois, riches en données, chacun d'eux a permis de mettre en lumière certains points particuliers:
- l'étude de la Durance a montré l'importance de la reconnaissance initiale de la rivière, du choix des soustronçons liés aux apports, de la bonne estimation des pourcentages de représentativité des faciès étudiés;

- l'étude de l'Orne a fait l'objet de deux approches: modélisation hydraulique classique complète d'un tronçon et modélisation simplifiée sur un nombre limité de transects pour un second tronçon; elle a de plus permis 
d'évaluer les incertitudes liées à la modélisation;

- l'étude du Lignon est un exemple où la modulation du débit réservé pourrait s'avérer très intéressante.

\section{V.1 La Durance à l'aval de Serre- Ponçon}

Le tronçon d'étude s'étend sur $10 \mathrm{~km}$ entre le réservoir d'Espinasses et le pont de l'Archidiacre (figure 9). Le débit réservé fixé par le cahier des charges étant de $1,5 \mathrm{~m}^{3} / \mathrm{s}$, pour un module de $80 \mathrm{~m}^{3} / \mathrm{s}$. Plusieurs demandes étaient formulées, en particulier par les pêcheurs de truites, pour relever ce débit à $2 \mathrm{~m}^{3} / \mathrm{s}, 6 \mathrm{~m}^{3} / \mathrm{s}$, et même pour les plus extrêmes à $12 \mathrm{~m}^{3} / \mathrm{s}$.
Une étude approfondie de l'état général de ce tronçon a été entreprise par le CEMAGREF Aix-en-Provence, le SRÁE, l'Université de Marseille et EDF-DER. L'Université de Marseille et la DER (Albignat et al., 1987) ont en particulier étudié l'habitat disponible pour les populations de truites.

\section{V.1.1 Les secteurs représentatifs et la reconnaissance du tronçon}

Quelques 22 transects répartis sur tout le tronçon ont fait l'objet de mesures détaillées pour trois débits (environ $1,5,3$ et $6 \mathrm{~m}^{3} / \mathrm{s}$ - mesures effectuées par la DER ou le cabinet CERIC). Une reconnaissance précise du tronçon (parcours à pied des $10 \mathrm{~km}$ ) a permis d'une part de déterminer le pourcentage de représenta-

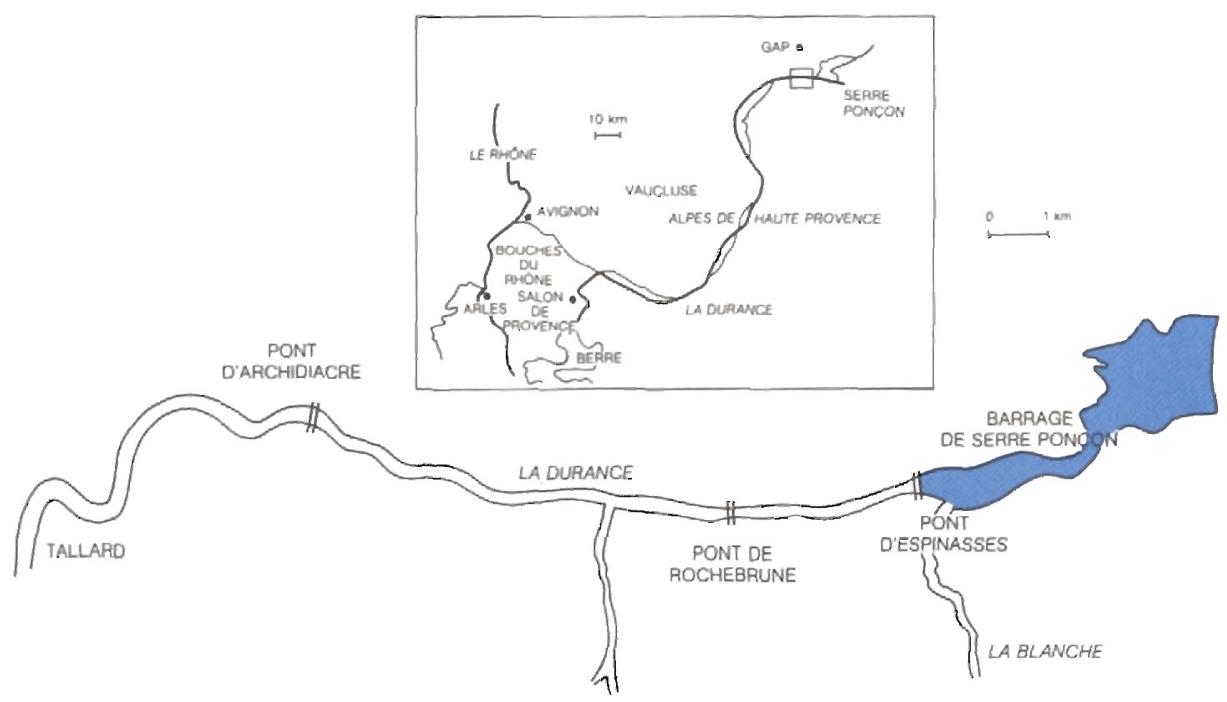

Fig. 9. - La Durance à l'aval de Serre-Ponçon - Plan de situation.

Fig. 9. - The Durance river downstream of Serre-Ponçon dam - General overview. 
tivité des différents types d'écoulement mesurés et de noter d'autre part de façon précise les zones colmatées ou envasées (afin d'affecter à ces zones une valeur d'habitat correspondant à un substrat effectivement colmaté). Ce colmatage et cet envasement, par endroits importants, sont dûs aux apports solides des affluents en périodes d'orages, les vitesses dans le lit principal étant trop faibles pour assurer le transport de toutes les matières en suspension à l'aval.

\section{Remarque:}

Le secteur étudié à été découpé en 4 sous-tronçons du fait de l'importance des apports intermédiaires: les débits transitant sont en effet pour un même débit réservé lâché au barrage, très différents d'un sous-tronçon à l'autre et d'une saison à l'autre.

\section{V.1.2 Les notes d'habitats obte- nues}

La figure 10 donne à titre d'exemple, les notes d'habitats obtenues sur l'ensemble du tronçon pour les 3 stades de développement de la truite en été (où les conditions de débit sont les plus contraignantes car les apports intermédiaires assez faibles sur les 4 secteurs d'étude).

\section{V.1.3 Synthèse des résultats et choix d'une gestion de la riviere}

Sur le strict plan de l'habitat piscicole, les résultats précédents ont montré que la Surface Pondérée Utile est pour l'adulte et pour l'ensemble du tronçon à $80 \%$ de l'optimum observé pour un débit réservé de $1,5 \mathrm{~m}^{3} / \mathrm{s}$ et à $90 \%$ à partir de $3 \mathrm{~m}^{3} / \mathrm{s}$. Pour le stade juvénile, elle augmente peu à partir de $1,5 \mathrm{~m}^{3} / \mathrm{s}$ (où elle vaut $92 \%$ du maximum observé). Elle décroît à partir de $5,5 \mathrm{~m}^{3} / \mathrm{s}$. Elle est pratiquement optimale dès $1 \mathrm{~m}^{3} / \mathrm{s}$ pour décroître à partir de $4,5 \mathrm{~m}^{3} / \mathrm{s}$ en ce qui concerne les alevins. Autrement dit, au delà d'un débit réservé d'environ $3 \mathrm{~m} / \mathrm{s}$, le gain d'habitat piscicole devient faible, voire négatif dans la gamme de débits étudiée.

En ce qui concerne les paramètres autres que l'habitat piscicole, les études ont montré que:

- la température n'était pas un paramètre limitant du fait du rejet d'une eau à $12^{\circ} \mathrm{C}$, d'apport de nappes également $12^{\circ} \mathrm{C}$, et de grands pools à forte inertie thermique qui constituent près de $50 \%$ du tronçon. Toutefois, un modèle a montré qu'il n'était pas souhaitable de maintenir le débit réservé en dessous de $1,5 \mathrm{~m}^{3} / \mathrm{s}$, afin d'éviter de trop fortes pointes journalières en été;

- l'oxygène dissous est satisfaisant pour les salmonidés, et en 2 ans de mesures, ce paramètre n'est jamais descendu en dessous de $6 \mathrm{mg} / \mathrm{l}$. Ceci malgré une abondante végétation fixée, dont on aurait ou craindre un impact négatif;

- les ressources trophiques mesurées ont montré qu'elles étaient altérées par le colmatage évoqué ci-dessus, mais qu'elles n'étaient pas limitantes pour les populations de truites;

- quelques pêches ont montré une biomasse inégalement répartie, mais assez importante dans certains secteurs (de 50 à $200 \mathrm{~kg} / \mathrm{ha}$ ); 


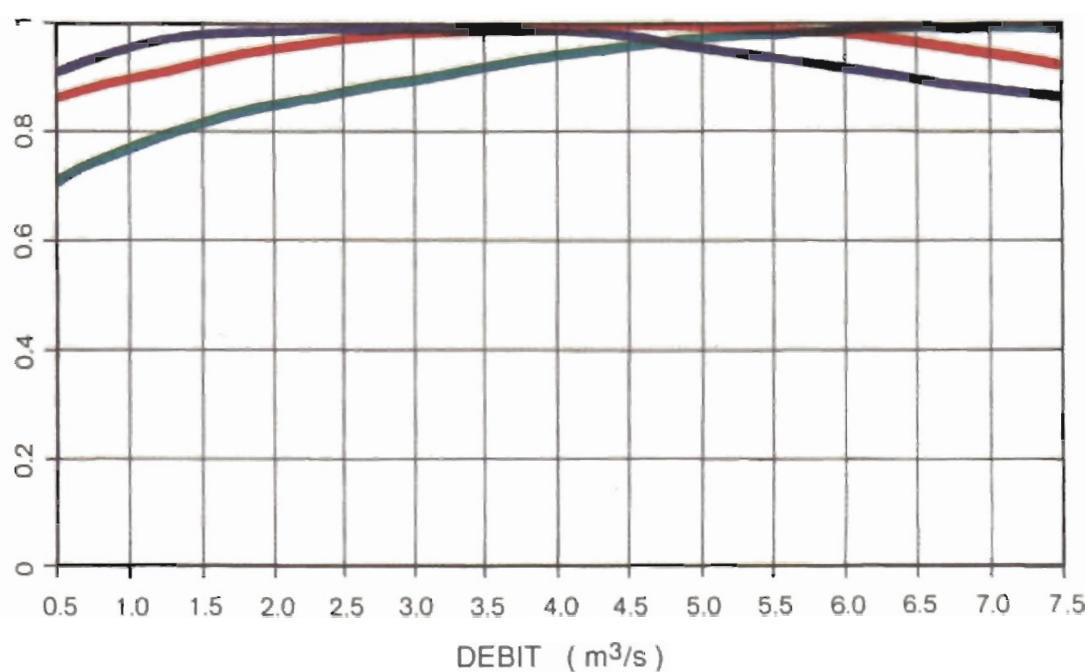

Fig. 10. - Courbes d'évolution de l'habitat obtenues sur la Durance pour la truite fario en fonction du débit lâché à l'aval de Serre Ponçon (SPU normée par rapport au maximum observé).

Fig. 10. - Habitat quality values in relation with the compensation discharge from Serre-Poncon reservoir for brown trout (Ratio of weighted usable area on macimum observed value).

- le lit est presque partout colmaté, ce qui peut être une gêne pour le frai, et en tout cas un désagrément pour la pêche et les loisirs nautiques. II en est de même en quelques endroits très envasés, ce qui altère directement la qualité de l'habitat;

Le "mal " de la Durance n'est pas tant en fait I'habitat hydraulique, ou la qualité physico-chimique, mais la sédimentation.

A partir de ces éléments, deux décisions ont pu être prises:

- relever le débit réservé à $2 \mathrm{~m}^{3} / \mathrm{s}$ (ce qui permet d'accroître jusqu'à $85 \%$ la capacité d'accueil du milieu pour l'adulte); tation (piégeage de sédiment, recalibrage écologique); en effet, il apparaît qu'au delà de $2 \mathrm{~m}^{3} / \mathrm{s}$ un aménagement "doux" du lit devrait être plus efficace sur l'entraînement des vases qu'une augmentation du débit réservé, dans la mesure où, pour la gamme de débits envisagée, les vitesses ne s'accroîtraient pas suffisamment pour empêcher le colmatage.

\section{V.2 L'Orne à l'aval de Rabodanges}

Un projet de réimplantation du saumon et de la truite de mer a été lancé par le Conseil Supérieur de la Pêche sur l'Orne entre la retenue de Rabo- 
poissons vient d'être réalisée). Ce secteur des Gorges de Saint Aubert représente l'objectif majeur du programme de restauration des migrateurs sur l'Orne engagé depuis 1981 et conforté par le contrat de plan EtatRégion (1989-1993). Le secteur de rivière concerné (figure 11) se compose de deux parties distinctes: un tronçon de $4,5 \mathrm{~km}$ à débit réservé et un tronçon de $5,5 \mathrm{~km}$ à l'aval de la restitution de l'usine soumis à de fréquentes éclusées.

Une étude a été effectuée en collaboration avec le CSP afin de déterminer une valeur de débit réservé à l'aval de Rabodanges adaptée aux besoins des deux espèces.

Parallèlement à un suivi de la qualité de l'eau, la qualité d'habitat a été étudiée pour les deux espèces. La méthode des micro-habitats a été appliquée par EDF (Courot, 1988) sur les deux tronçons. Des courbes de preferenda ont été établies pour le frai du saumon et de la truite de mer et pour les stades alevin et juvénile du saumon, à l'aide d'éléments trouvés dans la littérature et des estimations du CSP. Les courbes utilisées pour l'alevin et le juvénile de truite de mer sont celles de la truite fario pour ces mêmes stades (même espèce à ces périodes de vie).

\section{V.2.1 Les mesures effectuées}

Le Conseil Supérieur de la Pêche a effectué le long de transects des mesures de vitesses, profondeurs et substrat, à raison de 4 points par transect et d'un transect tous les 100 mètres sur les 10 kilomètres de ri- vière étudiés et ce pour 4 débits lâchés $\left(0,21-0,43-0,7-\right.$ et $\left.1 \mathrm{~m}^{3} / \mathrm{s}\right)$. Ces données ont servi au CSP pour mettre en œuvre une approche très voisine de celle des micro-habitats.

Le protocole de mesures et de modélisation employé par la DER fut différent selon le tronçon étudié.

\section{- Tronçon à éclusées}

Sur ce tronçon à plus faible pente, les conditions d'écoulement régulières ont permis d'utiliser un modèle de ligne d'eau en écoulement permanent pour calculer l'évolution des paramètres hydrauliques en fonction du débit de chacun des 55 transects mesurés par le CSP. Quatre verticales par transect pouvant s'avérer insuffisantes, sept transects ont été étudiés plus finement ( 1 verticale tous les mètres) pour reconstituer des profils plus précis et caler ainsi le modèle hydraulique sur l'ensemble des 55 sections.

Les notes d'habitat du tronçon à éclusées, calculées en fonction d'un débit "de base" hors éclusées, ont alors été estimées à partir des vitesses, profondeurs et substrat de ces 55 transects (chacun étant supposé représentatif de 100 mètres de rivière).

\section{- Tronçon à débit réservé}

Sur ce tronçon où le substrat est très chaotique, la modélisation hydraulique classique s'est avérée impossible.

Une reconnaissance complète, assortie de mesures ponctuelles de vitesses et profondeurs a été effectuée. Une analyse de données a permis de retenir les 9 transects (parmi ceux du $\mathrm{CSP}$ ) les plus caractéristiques. 

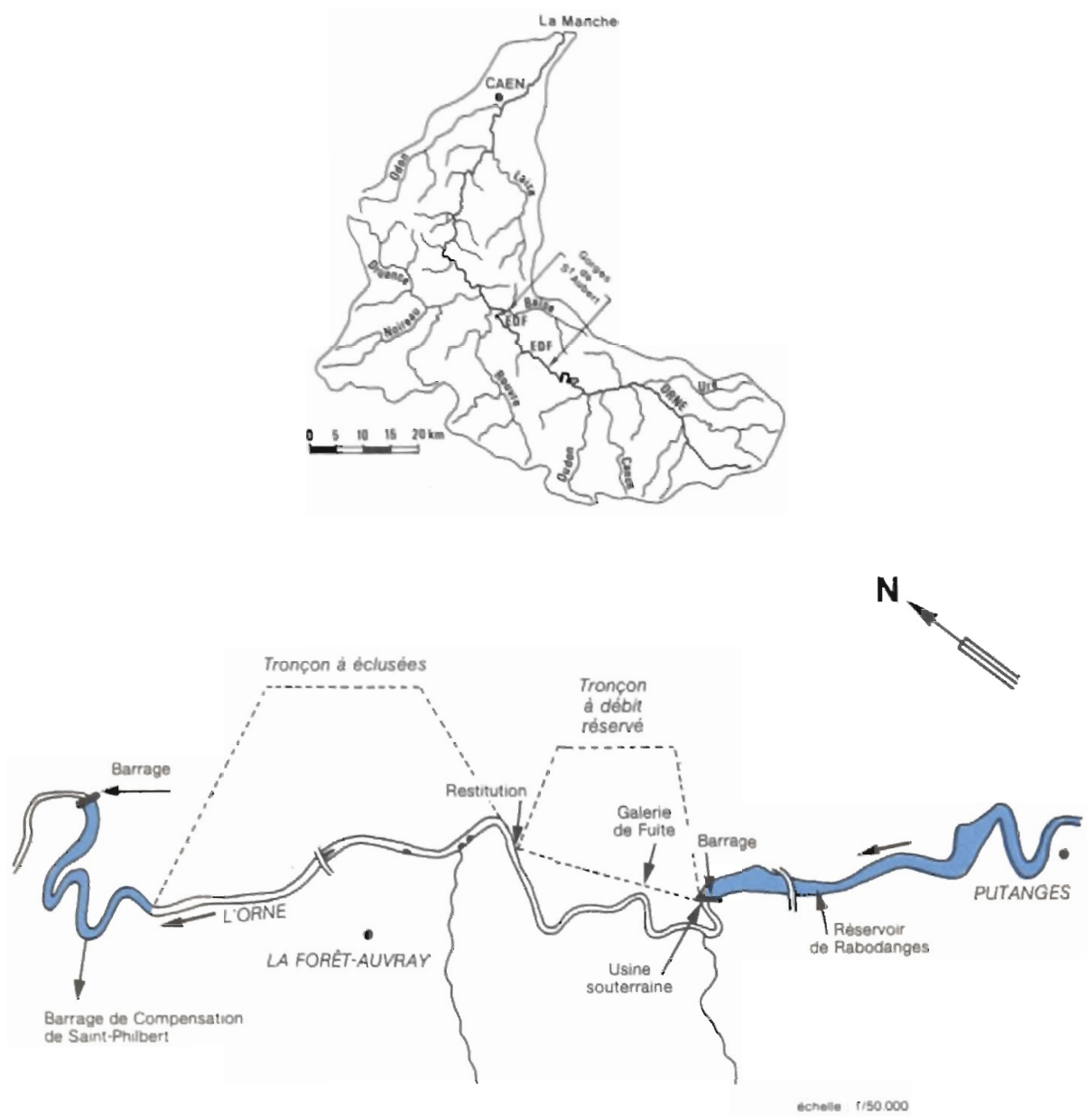

Fig. 11. - L'Orne à l'aval de Rabodanges - Plan de situation.

Fig. 11. - The Orne river downstream from Rabodanges reservoir - General overview.

Les notes d'habitat du tronçon ont alors été obtenues selon la démarche présentée § IV.3 et IV.4.

\section{V.2.2 Les notes d'habitat obte- nues}

Les figures 12 et 13 présentent l'évolution avec le débit réservé des Sur- faces Pondérées Utiles des 2 tronçons pour le saumon et la truite de mer.

Le tableau présenté figure 8 résume les résultats obtenus sur l'ensemble des 2 tronçons pour les deux espèces. 

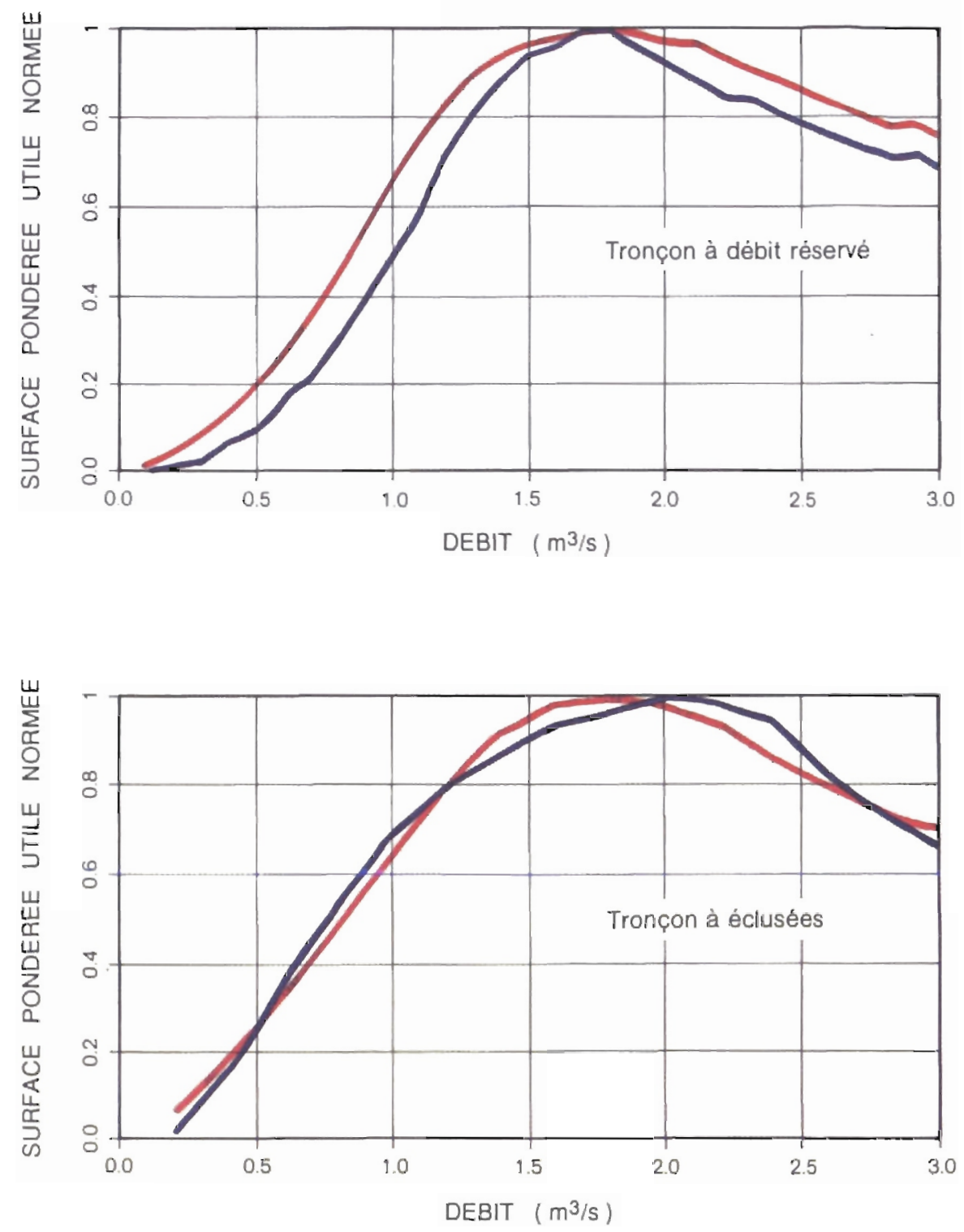

Fig. 12. - Courbes d'évolution de l'habitat obtenues sur l'Orne à l'aval de Rabodanges pour le saumon (SPU normée par rapport au maximum observé).

Fig. 12. - Habitat quality values in relation with the compensation discharge from Rabodanges reservoir for salmon (ratio of Weighted Usable Area on maximum observed value). 

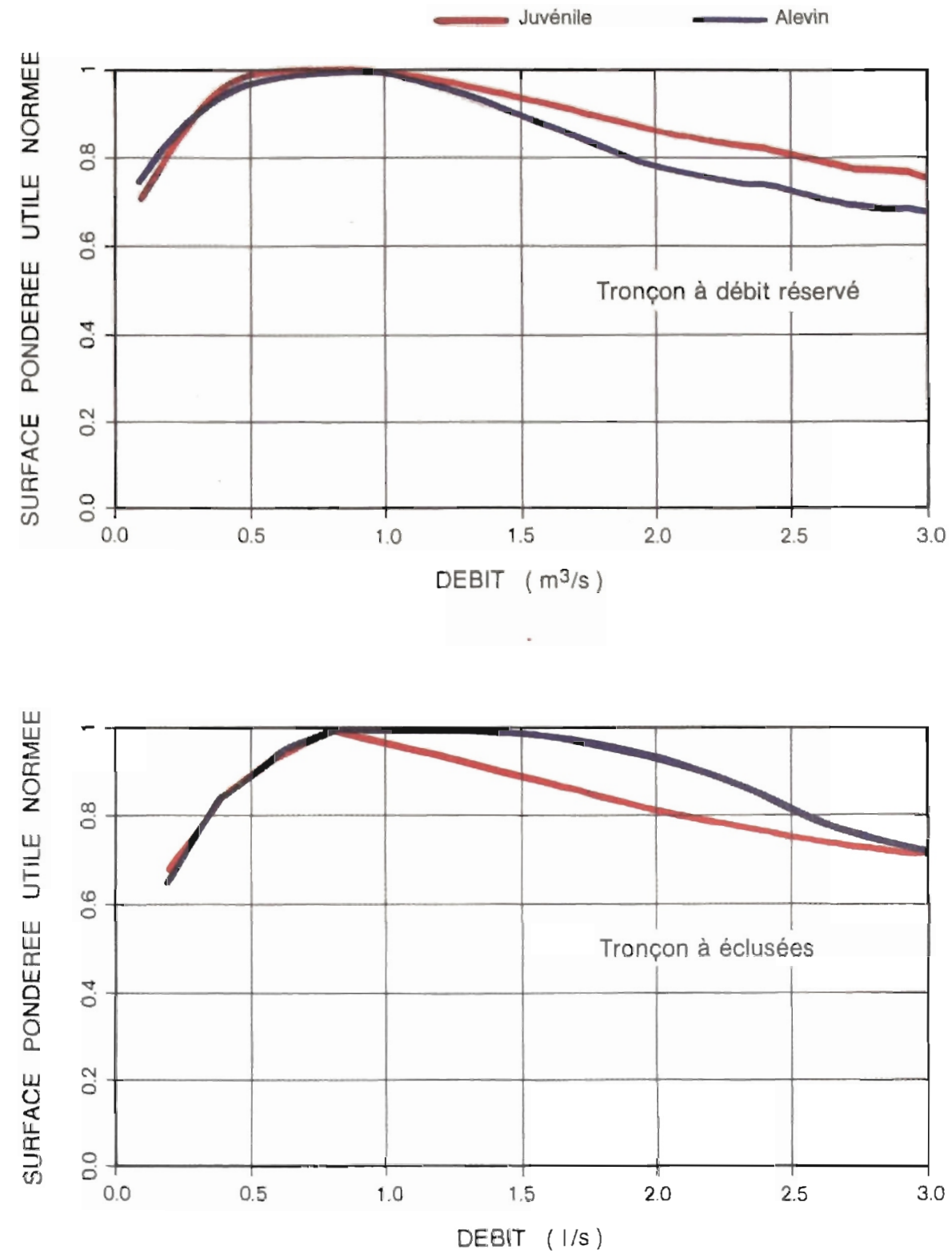

Fig. 13. - Courbes d'évolution de l'habitat obtenues sur l'Orne à l'aval de Rabodanges pour la truite de mer (SPU normée par rapport au maximum observé).

Fig. 13. - Habitat quality values in relation with the compensation discharge from Rabodanges reservoir for sea trout (ratio of Weighted Usable Area on maximum observed value). 


\section{V.2.3 Synthèse des résultats et choix d'un débit réservé}

La truite de mer est une espèce dont les besoins sont différents suivants les stades. Alors que le débit réservé imposé par la loi Pêche $\left(0,2 \mathrm{~m}^{3} / \mathrm{s}\right)$ convient aux juvéniles et aux alevins, la reproduction nécessite un débit nettement plus élevé (au moins 1,2 $\mathrm{m}^{3} / \mathrm{s}$ pour l'optimum et $0,8 \mathrm{~m}^{3} / \mathrm{s}$ pour des conditions favorables).

Le saumon demande de fortes valeurs de débit toute l'année. Le stade le plus exigeant est l'alevin: il faut au minimum $1 \mathrm{~m}^{3} / \mathrm{s}$ pour avoir un habitat favorable et $1,3 \mathrm{~m}^{3} / \mathrm{s}$ pour un habitat optimum.

Cette étude a montré, dans ce cas particulier, l'insuffisance du débit conforme à la loi pêche $(200 \mathrm{l} / \mathrm{s}$ soit le $1 / 40^{\mathrm{e}}$ du module) pour la réimplantation des deux espèces. Dans le cadre d'une convention avec le Ministère de l'Environnement et avec le soutien financier des collectivités locales - départements et région -, un débit expérimental de $800 \mathrm{l} / \mathrm{s}$ (minimum favorable pour la presque totalité des stades) a été mis en place par EDF; un réalevinage en jeunes saumons assorti d'un suivi de la recolonisation et des différents compartiments de l'écosystème complète cette mesure. Un problème de qualité d'eau semble en particulier se poser à l'aval immédiat du barrage : les eaux restituées sont mal oxygénées et les deux premiers kilomètres présentent des teneurs assez faibles en oxygène dissous depuis le passage de 200 à $800 \mathrm{l} / \mathrm{s}$ (ce phénomène n'existant pas à $200 \mathrm{l} / \mathrm{s}$, grâce à l'apport des eaux de bonne qualité d'un petit affluent qui devient négligeable par rapport aux $800 \mathrm{l} / \mathrm{s}$ délivrés). II s'agit également de suivre l'impact effectif des éclusées sur la reproduction et les jeunes alevins: l'étude de l'habitat du tronçon à éclusées n'a tenu compte que du débit minimum hors éclusées, et il faut être conscient du fait que ce n'est pas forcément ce régime minimum qui est le plus limitant.

\section{V.3 Le Lignon du Velay à l'aval de Lavalette}

L'étude du Lignon (Massif Central), entre Lavalette et l'usine de Vendet (figure 14) a été entreprise dans le cadre d'un contrat de rivière. L'étude des habitats piscicoles a été menée conjointement par EDF et le CEMAGREF (Souchon et al., 1990) afin de comparer les deux types d'approches mis en œuvre (mesures/modélisation simplifiée pour EDF - mesures/modélisation classique pour le CEMAGREF).

\section{V.3.1 Reconnaissance de la ri- vière et choix des faciès repré- sentatifs}

Les accès à la rivière étant particulièrement malaisés, et la longueur importante $(17 \mathrm{~km})$, un survol par hélicoptère avec prise de vue vidéo s'est avéré particulièrement précieux.

Le Lignon s'écoule dans le Velay granitique, en zone volcanique avec socle cristallin. Le module est de $6 \mathrm{~m}^{3} / \mathrm{s}$ et le débit moyen en Août est de $1,5 \mathrm{~m}^{3} / \mathrm{s}$. Le débit réservé dans le tronçon court-circuité est de $200 \mathrm{l} / \mathrm{s}$, 
Secteur de Mesure

USINE DE VENDETS
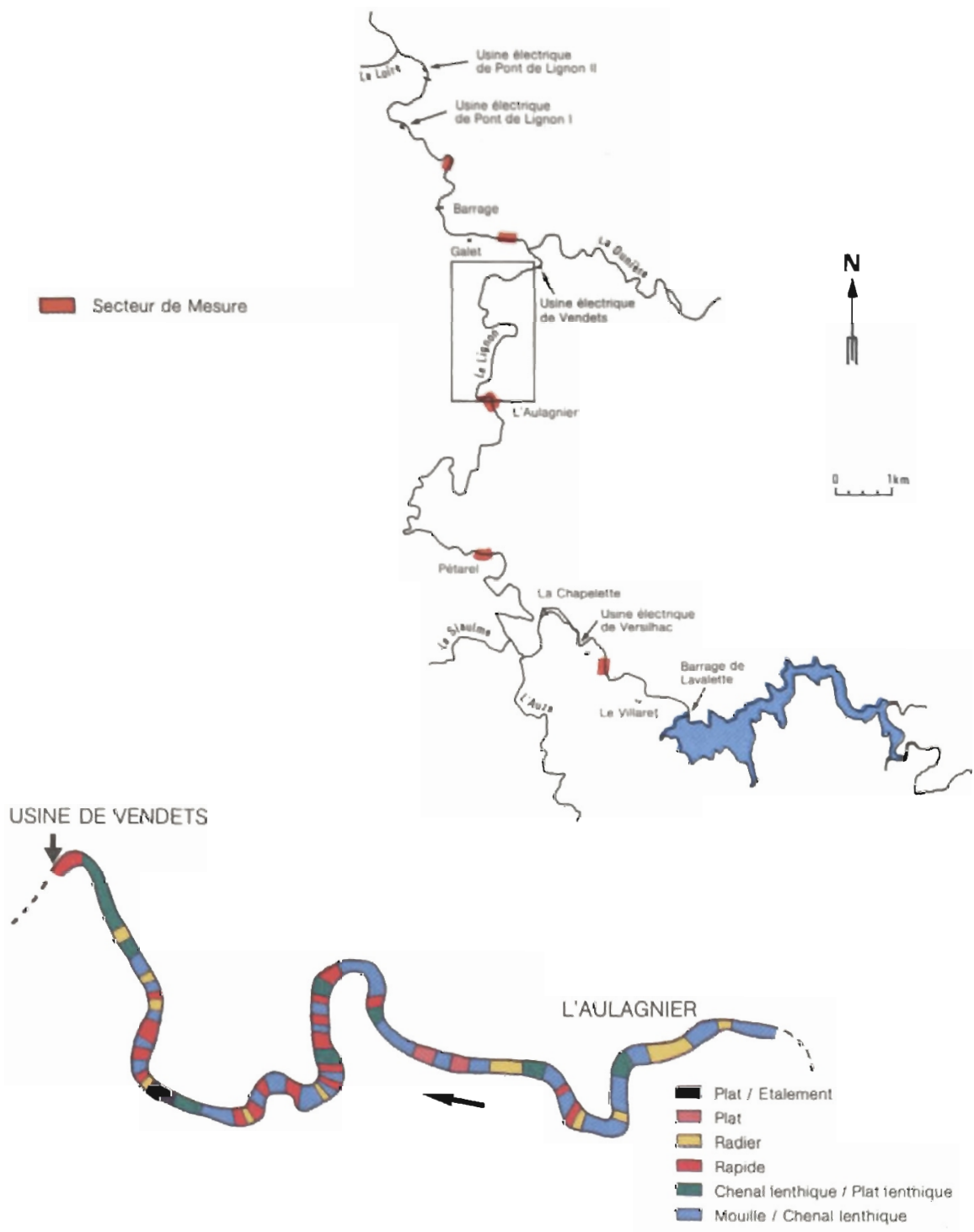

(d'apres Malavai et/ou Chaveroche)

Fig. 14. - Le Lignon - Plan de situation et exemple de découpage en faciès.

Fig. 14. - The Lignon river - General overview and exemple of division into "facies". 
auquel s'ajoute tout à l'amont les apports intermédiaires de l'Auze et de la Siaulme.

La rivière a été sectorisée en faciès (figure 14); elle est constituée d'environ $9 \%$ de rapides, $17 \%$ de radiers, $58 \%$ de pools et $16 \%$ de plats.

Les faciès représentatif́s ont été choisis dans cinq secteurs (au niveau de Villaret, Pétarel, Aulagnier, Galets et Cublaise) dans des biefs soumis à différents rythmes hydrologiques.

Les mesures ont été réalisées dans 19 faciès, à raison d'un transect par faciès, et pour deux débits réservés extrêmes: $0,2 \mathrm{~m}^{3} / \mathrm{s}$ et $1 \mathrm{~m}^{3} / \mathrm{s}$.

\section{Remarque:}

II faut noter ici que les études d'incertitude faites ultérieurement ont montré que cette quantité d'information était insuffisante, et qu'il aurait fallu réaliser en fait 2 à 3 transects par faciès, et ceci pour 3 débits (ajouter un débit intermédiaire vers $0,6 \mathrm{~m}^{3} / \mathrm{s}$ ).

\section{V.3.2 Les Notes d'habitat obte- nues}

Les résultats obtenus par les deux équipes sont suffisamment concordants pour aider à la prise de décision.

La figure 15 reproduit les courbes d'évolution de la qualité d'habitat obtenues par EDF pour chacun des secteurs et pour l'ensemble du tronçon.

II apparaît clairement que, du point de vue de l'habitat hydraulique, la situation actuelle se situe déjà globalement pour l'ensemble du tronçon dans la plage la meilleure, et qu'un léger gain pourrait éventuellement être obtenu jusqu'à un débit de $0,6 \mathrm{~m}^{3} / \mathrm{s}$.

Il faut toutefois noter que pour certains secteurs particuliers tels que ceux représentés par les stations de Villaret et d'Aulagnier, une valeur de débit de l'ordre de 400 à $500 \mathrm{l} / \mathrm{s}$ permettrait de se situer à plus de $80 \%$ de l'optimum pour l'ensemble des stades (contre environ $70 \%$ à $200 \mathrm{l} / \mathrm{s}$ pour certains autres).

\section{V.3.3 Synthèse et choix d'un mode de gestion}

Dans le contrat de rivière Lignon, l'objectif piscicole a été souligné avec affichage d'une vocation salmonicole pour tout le secteur d'étude.

Dans l'étude globale du tronçon, les résultats de relativement bonnes qualités d'habitat obtenus dès $200 \mathrm{l} / \mathrm{s}$ s'expliquent en partie par les apports des affluents $(+200 \mathrm{l} / \mathrm{s}$ pendant les mesures).

Une analyse détaillée de ces apports (Rietjens, 1990) a permis de reconstituer au cours de l'année les débits effectifs moyens transitant dans la rivière en fonction du débit réservé. L'importance de ces apports en certaines périodes de l'année laisse apparaître clairement l'intérêt d'une modulation du débit.

A titre d'exemple la figure 16 montre l'évolution au cours d'une année sèche de la Surface Pondérée Utile pour la truite adulte à la station dite "Aulagnier" - pour laquelle le gain en habitat est le plus sensible lors 


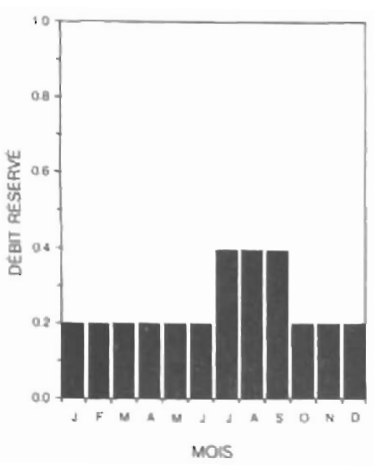

Scénario 1

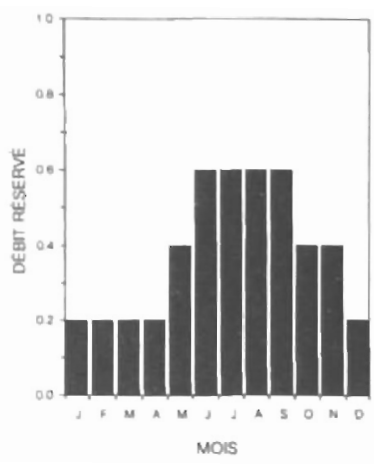

Scénario 2

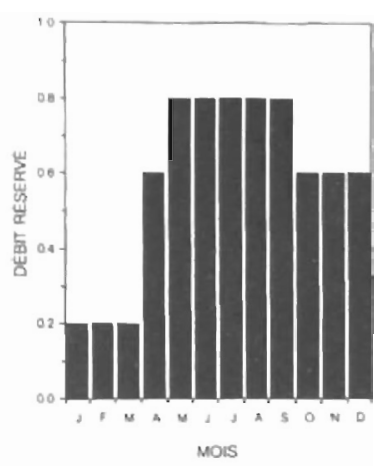

Scénario 3

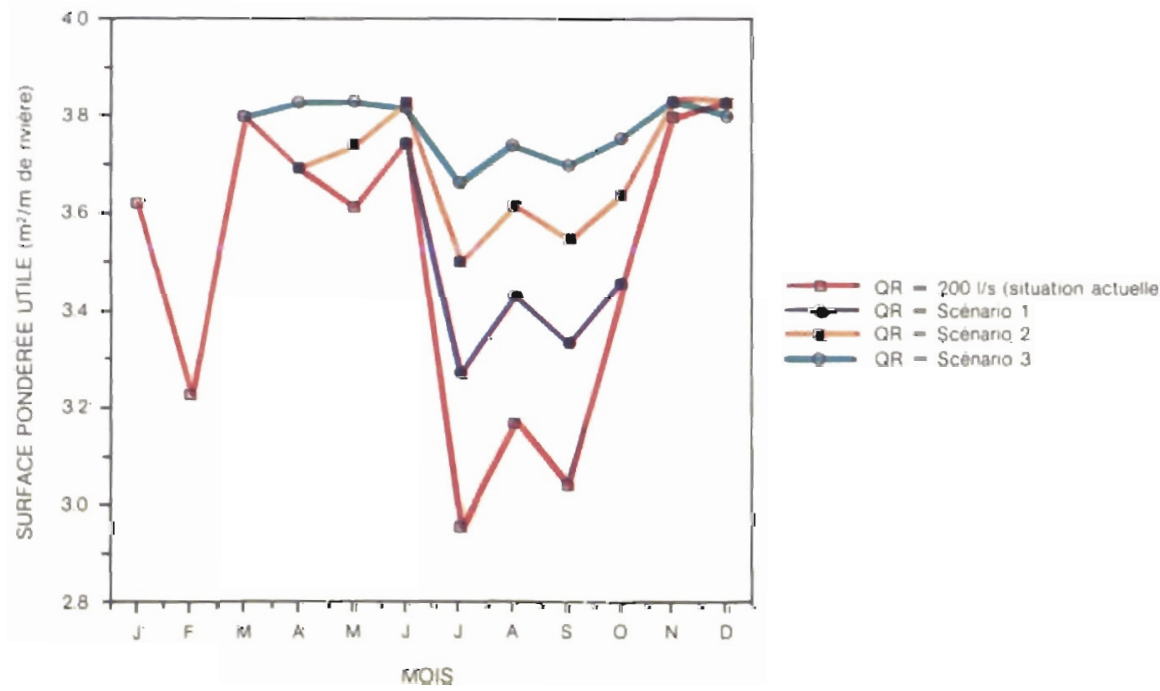

Fìg. 16. - Chronologies de la qualité d'habitat pour la truite fario sur le Lignon obtenues pour 3 scénarii possibles de gestion - année sèche - station Aulagnier.

Fig. 16. - Time series ofhabitat quality value for brown trout on the Lignon river computed for three possible management scenarios - dry year - Aulagnier sector. 
d'une augmentation de débit - dans trois scénarii possibles de gestion.

On voit nettement, en cette station, l'intérêt d'augmenter le débit réservé pour l'adulte en période estivale exclusivement: intérêt écologique et intérêt économique collectif peuvent fort bien cohabiter.

\section{CONCLUSION}

Après 10 ans de recherches, d'expérimentation in situ, puis de mise en œuvre de la méthode des microhabitats, le temps est venu d'en faire un bilan.

En premier lieu, la validité de ses principes est maintenant établie: les résultats des études faites en France convergent avec ceux des chercheurs américains, et permettent de confirmer le bien fondé de la notion de préférence d'habitat qui est à la base de la méthode. Un étalonnage des courbes de préférence de la truite des cours d'eau français a été réalisé, et ces courbes s'avèrent peu différentes des courbes américaines.

On dispose ensuite, ce qui est fondamental pour la fiabilité des résultats, d'un protocole de mise en œuvre garantissant une bonne utilisation de la méthode et une bonne estimation des notes de qualité d'habitat. L'approche "par faciès" de la reconnaissance de la rivière s'est avérée bien adaptée et assez objective pour un expérimentateur averti. La première classification des faciès proposée permet de décrire rapidement la morphologie de la rivière. On connaît enfin un bon ordre de grandeur des mesures à effectuer dans les faciès représentatifs du tronçon étudié pour obtenir une précision acceptable.

L'outil d'aide à la décision est maintenant opérationnel. II convient de l'utiliser et de l'intégrer plus systématiquement dans les études de sites où l'enjeu piscicole est présent. L'utilisation d'un protocole "standardisé" (estimation sans modèle hydraulique basée sur des mesures à 4 débits au minimum, calculs effectués à l'aide d'un logiciel convivial...) devrait permettre, dans le cas des études d'impact et pour des sites pas trop complexes, d'en réduire le coût unitaire.

Pour conclure, il est clair que la méthode des micro-habitats apporte une information technique qui constitue une aide précieuse à la décision; elle permet notamment d'estimer s'il y a peu ou beaucoup à attendre d'un changement éventuel de débit.

II faut noter toutefois que cette méthode ne constitue pas elle-même une règle de choix de débit. Ce choix est directement lié aux types d'objectifs fixés, que ce soit dans le cadre réglementaire de la loi pêche par exemple, ou dans un cadre plus général d'une gestion de rivière: cherche-t-on à obtenir une biomasse maximale de poissons ou bien assurer un certain équilibre des populations? Veut-on améliorer l'ensemble d'un tronçon, ou bien privilégier certains secteurs particuliers? S'agit-il de développer des usages récréatifs comme la pêche ou de se rapprocher d'une situation antérieure, parfois dite "naturelle"? 
Faute d'objectifs clairement définis, et c'est par exemple le cas de la loi pêche - "garantir en permanence la vie, la circulation et la reproduction des espèces..." -, l'analyse des résultats donnés par le modèle ne peut que dépendre de l'expert chargé de proposer un choix de débit.

Des efforts vers la définition de critères objectifs doivent donc être maintenant entrepris pour pouvoir utiliser au mieux cet outil de gestion piscicole.

\section{REMERCIEMENTS}

Cet article a pu être écrit grâce aux travaux de nombreux chercheurs et techniciens - sans oublier de nombreux stagiaires -, qu'ils appartiennent à EDF, à I'ENSA Toulouse, au CEMAGREF, aux Universités de Marseille et de Toulouse, au Conseil Supérieur de la pêche, à des Fédérations de Pêche. ciés.

Qu'ils soient tous sincerement remer-

\section{RÉFÉRENCES BIBLIOGRAPHIQUES}

Albignat J.P., Brun G., Sabaton C., 1987. Etude de l'évolution de l'habitat piscicole en Durance en fonction du débit lâché à Espinasses. Rapport EDF HE.31/87-12, $21 \mathrm{p}$.

Baume J.P., Poirson M., 1984. Modélisation numérique d'un écoulement permanent dans un réseau hydraulique maillé à surface libre, en régime fluvial. La Houille Blanche $n^{\circ} 1 / 2$.

Belaud A., Chaveroche P., Lim P., Sabaton C., 1989. Probability-of-use curves applied to brown trout (Salmo trutta fario L.) in rivers of southern France Regu- lated Rivers: Research \& Management, Vol. 3, 321-336.

Bovee K.D., 1983. A users guide to the Instream Flow Incremental Methodology. FWS/OBS-8320. U.S. Fish Wild. Serv., Coop. Instream Flow. Serv. Grp, Fort Collins, CO. $248 \mathrm{p}$.

CEMAGREF, 1988. Relation entre la biomasse piscicole d'un cours d'eau et sa valeur d'habitat. Convention EDF $n^{\circ} 2 F 6445$. Laboratoire d'Hydroécologie Quantitative. Lyon. $21 \mathrm{p}$.

Chaveroche P., Sabaton C., 1989. An analysis of brown trout (Salmo trutta fario L.) habitat. The role of qualitative data from expert advice in formulating probability-of-use curves. Regulated Rivers: Research \& Management, Vol. 3, 305-319.

Chaveroche P., 1990. Recherche sur les préférences d'habitat de la truite fario (Salmo trutta fario L.) (Aide à la détermination de la valeur d'un débit réservé). Thèse de Doctorat de I'Université. Université de Provence. $214 \mathrm{p}$.

Cherubini P., Lebosse A., 1992. Code de calcul d'écoulement en surface libre filaire. "Lido - Sara - Rezo". Version 2.0. Note de principe. Rapport EDF HE.43/92-64.

Courot A., 1988. Débit réservé de l'Orne à Rabodanges. Evaluation de la qualité d'habitat piscicole en fonction du débit. Rapport EDF HE.31/88-07. 23 p.

Courot A., 1989. Determination of Hydraulic Parameters for Instream Flow Assessments. Regulated Rivers: Research \& Management, Vol. 3, 337344.

Fragnoud E., 1987. Préférences d'habitat de la truite fario (Salmo trutta fario L., 1758) en rivière (quelques cours d'eau du Sud-Est de la France). These de Doctorat de I'Université. Université Claude Bernard - Lyon 1. $314 \mathrm{p}$.

Malavoi J.R., 1989. Typologie des Faciès d'écoulement ou unités morphodynamiques des cours d'eau à haute éner- 
gie. CEMAGREF. Groupement de Lyon. Division Qualité des Eaux, Pêche et Pisciculture - $20 \mathrm{p}$.

Parent A., 1991. Etude de protocoles expérimentaux d'analyses de la qualité d'habitat piscicole d'un tronçon de rivière à débit réservé. Rapport EDF HE.31/91-05 - $51 p$.

Rietjens J., 1990. Etude hydrologique de faisabilite d'une modulation du débit réservé du Lignon du Velay. Rapport EDF HE.31/90-33. 47 p.

Sabaton C., 1991. Quel débit réserver à l'aval des ouvrages hydroélectriques? La méthode des micro-habitats: un outil d'aide à la décision. Rapport EDF HE.31/91-26. 62 p.

Souchon Y., Trocherie F., Fragnoud E., Lacombe C., 1989. Les modèles numériques des micro-habitats des poissons: application et nouveaux développements. Revue des Sciences de l'eau. 2, 807-830.

Souchon Y., Malavoi J.R., Courot A., Miquel J., 1990. Le Lignon du Velay au barrage de Lavalette jusqu'au confluent de la Loire. Simulation de l'habitat des poissons. Aide à la détermination du débit résené. Rapport CEMAGREF/EDF/SRAE Auvergne. $60 \mathrm{p}$. 